CONFORMAL GEOMETRY AND DYNAMICS

An Electronic Journal of the American Mathematical Society

Volume 2, Pages 56-88 (August 19, 1998)

S $1088-4173(98) 00022-8$

\title{
RELATIVELY AND INNER UNIFORM DOMAINS
}

\author{
JUSSI VÄISÄLÄ
}

\begin{abstract}
We generalize the concept of a uniform domain in Banach spaces into two directions. (1) The ordinary metric $d$ of a domain is replaced by a metric $e \geq d$, in particular, by the inner metric of the domain. (2) The uniformity condition is supposed to hold only for certain pairs of points of the domain. We consider neargeodesics and solid arcs in these domains. Applications to the boundary behavior of quasiconformal maps are given. In particular, we study maps between domains of the form $E \times B$, where $E$ is a Banach space and $B$ is a ball.
\end{abstract}

\section{INTRODUCTION}

1.1. Uniform domains in euclidean $n$-space $\mathrm{R}^{n}$ were introduced by $\mathrm{O}$. Martio and J. Sarvas [MaS] in 1979, and independently by P.W. Jones [Jo1]. The notion was generalized for domains in Banach spaces by the author [Vä6]. There are plenty of equivalent characterizations for uniform domains. We recall the definition based on the quasihyperbolic metric. The alternative geometric approach will be considered in 2.16 .

Let $E$ be a real Banach space with $\operatorname{dim} E \geq 2$, and let $G \varsubsetneqq E$ be a domain. For $x \in G$ we let $\delta(x)=\delta_{G}(x)$ denote the distance $d(x, \partial G)$ between $x$ and the boundary $\partial G$ of $G$. The quasihyperbolic (QH) length of a rectifiable $\operatorname{arc} \gamma \subset G$ is the line integral

$$
l_{k}(\gamma)=\int_{\gamma} \frac{|d x|}{\delta(x)} .
$$

The $\mathrm{QH}$ distance between points $a, b \in G$ is

$$
k(a, b)=k_{G}(a, b)=\inf l_{k}(\gamma)
$$

over all rectifiable $\operatorname{arcs} \gamma$ joining $a$ and $b$ in $G$. The function $k=k_{G}$ is the $\mathrm{QH}$ metric of $G$.

For real numbers $r, s$ we use the notation:

$$
r \wedge s=\min (r, s), \quad r \vee s=\max (r, s) .
$$

For $a, b \in G$ we write

$$
r_{G}(a, b)=\frac{|a-b|}{\delta(a) \wedge \delta(b)}, \quad j_{G}(a, b)=\log \left(1+r_{G}(a, b)\right) .
$$

Received by the editors September 18, 1997 and, in revised form, April 14, 1998. 1991 Mathematics Subject Classification. Primary 30C65.

(C)1998 American Mathematical Society 
By a basic estimate for the QH metric [GP, 2.1], [Vä5, 2.2(1)], we have always

$$
j_{G}(a, b) \leq k_{G}(a, b) .
$$

Let $c \geq 1$. The domain $G$ is said to be quasihyperbolically (QH) c-uniform if

$$
k_{G}(a, b) \leq c j_{G}(a, b)
$$

for all $a, b \in G$.

Replacing the distance $|a-b|$ in (1.4) by the inner length distance

$$
\lambda(a, b)=\lambda_{G}(a, b)=\inf l(\gamma)
$$

over all $\operatorname{arcs} \gamma$ joining $a$ and $b$ in $G$, we obtain a larger class of domains, called $\mathrm{QH}$ inner c-uniform domains. These have been recently considered by Z. Balogh and A. Volberg [BV1],[BV2], and by M. Bonk, J. Heinonen and P. Koskela [BHK]. In [BV1], these domains are called uniformly John domains, and the definition is slightly different from the above; see 3.13.

In order to handle these and some other properties simultaneously, we assume that $e$ is a metric of $G$ such that

$$
|a-b| \leq e(a, b) \leq \lambda_{G}(a, b)
$$

for all $a, b \in G$. We say that $G$ is $\mathrm{QH}(c, e)$-uniform if

$$
k_{G}(a, b) \leq c j_{G}(a, b ; e)
$$

for all $a, b \in G$, where

$$
j_{G}(a, b ; e)=\log \left(1+r_{G}(a, b ; e)\right), \quad r_{G}(a, b ; e)=\frac{e(a, b)}{\delta(a) \wedge \delta(b)} .
$$

The case $e(a, b)=|a-b|$ gives ordinary $\mathrm{QH}$-uniform domains; the case $e=\lambda_{G}$ gives the $\mathrm{QH}$ inner $c$-uniform domains.

Throughout the paper, $e$ will be a metric of $G$ satisfying (1.8) (unless $e=$ $2.718281 \ldots$ in obvious cases). For example, $e$ may be the inner diameter metric $\varrho_{G}$, defined by

$$
\varrho_{G}(a, b)=\inf d(\gamma)
$$

over all arcs $\gamma$ joining $a$ and $b$ in $G$; here $d(\gamma)$ is the diameter of $\gamma$ in the norm metric. If $e \leq e^{\prime}$ are two metrics, then every $\mathrm{QH}(c, e)$-uniform domain is trivially $\mathrm{QH}\left(c, e^{\prime}\right)$-uniform.

For example, balls and half spaces are $\mathrm{QH} c$-uniform with a universal $c$. A disk in the plane with a radial slit is $\mathrm{QH}$ inner uniform but not $\mathrm{QH}$ uniform. A parallel strip in the plane is not $\mathrm{QH}$ inner uniform.

In this paper we consider relative versions of uniformity and inner uniformity. If $A \subset G$ and if (1.6) holds for all $a, b \in A$, we say that $G$ is $\mathrm{QH} c$-uniform relative to $A$, or briefly, QH $c$-uniform rel $A$. If $R>0$ and if $G$ is $\mathrm{QH} c$-uniform rel $A$ for all $A \subset G$ with diameter $d(A) \leq R$, we say that $G$ is $R$-boundedly $\mathrm{QH}$ c-uniform. Equivalently, this means that (1.6) holds whenever $a, b \in G$ and $|a-b| \leq R$.

Following the usual practice, we omit the parameters in this and later terminology if we do not want to specify their values. For example, a domain is boundedly QH uniform if it is $R$-boundedly QH $c$-uniform for some $R$ and $c$.

The corresponding relative $\mathrm{QH}$ inner and $(c, e)$-uniformity properties are defined in the obvious way. For example, $G$ is $R$-boundedly $(c, e)$-uniform if (1.9) holds whenever $e(a, b) \leq R$. 
For example, a parallel strip in $\mathrm{R}^{2}$ is $R$-boundedly $\mathrm{QH}$-uniform with some constant $c=c_{R}$ for all $R>0$. More generally, let $E_{1}$ and $E_{2}$ be Banach spaces and let $B_{2}$ be the unit ball of $E_{2}$. Then the domain $G_{0}=E_{1} \times B_{2}$ is $R$-boundedly QH $c_{R}$-uniform for all $R>0$.

The purpose of this paper is to develop a theory of relatively and boundedly uniform and inner uniform domains. In particular, we study neargeodesics and solid arcs in these domains.

The general theory of uniform domains and their generalizations in a Banach space $E$ is given in Section 2, and the special case $E=R^{n}$ is considered in Section 3. Since I hope that these sections can be used in the future as a "handbook" on uniform domains and their generalizations, the results are given in a rather general form. We also give in 2.42 a new characterization of uniform domains, based on the invariance of neargeodesics under domain enlarging. Section 4 deals with coarsely quasihyperbolic maps $f: G \rightarrow G^{\prime}$; the definition is recalled in 4.2. In the case $E=R^{n}$, this class contains all quasiconformal maps and also the pseudo-isometries of Thurston [Th, 5.9]. The main result 4.8 gives a sufficient condition for $f$ to have a limit at a point $b \in \partial G$. In Section 5 we consider the special case where the domain is of the form $G_{0}=E_{1} \times B_{2}$. For example, if $f: G_{0} \rightarrow G_{0}$ is $C$-coarsely $M$-QH and if $\operatorname{dim} E_{1} \geq 2$, then $f$ extends to a homeomorphism $\bar{f}: \bar{G}_{0} \rightarrow \bar{G}_{0}$ with $\bar{f}(\infty)=\infty$. Moreover, $\bar{f}$ is quasisymmetric rel $\partial G_{0}$, and the horizontal and the vertical distortions of $f$ are controlled by $(M, C)$.

As a special case we get results on $K$-quasiconformal maps of $G_{0}=\mathrm{R}^{p} \times B^{q} \subset$ $\mathrm{R}^{p+q}$. Alternatively, these results could be obtained by using moduli of suitable path families. However, the author believes that in the theory of quasiconformal maps, one should try to find alternative proofs for various results by replacing the path families by quasihyperbolic arguments, since this would often give stronger results, where (1) quasiconformality is replaced by solidity or by coarse quasihyperbolicity and (2) $\mathrm{R}^{n}$ is replaced by an arbitrary Banach space.

The basic theory of uniform and relatively uniform domains is valid in all normed spaces. However, in the applications to the boundary behavior of maps, the completeness of the space is essential.

1.12. Notation. Throughout the paper, $E$ will denote a real Banach space with $\operatorname{dim} E \geq 2$, and $G$ is always a proper subdomain of $E$. The norm of a vector $x \in E$ is written as $|x|$. For balls and spheres we use the fairly standard notation

$$
\begin{gathered}
B(a, r)=\{x:|x-a|<r\}, \quad \bar{B}(a, r)=\{x:|x-a| \leq r\}, \\
S(a, r)=\{x:|x-a|=r\} .
\end{gathered}
$$

The center $a$ can be omitted if it is the origin. In particular, $B(1)$ is the open unit ball of $E$.

By an arc we mean a set homeomorphic to a real interval, which may be closed, open, or half open. We write $\gamma: a \curvearrowright b$ if $\gamma$ is an arc with endpoints $a$ and $b$. This notation also gives an orientation for $\gamma$ such that $a$ is the first point. If $x$ and $y$ are points of an arc $\gamma$, we let $\gamma[x, y]$ denote the compact subarc of $\gamma$ between $x$ and $y$. For half open subarcs we use the obvious notation $\gamma[x, y)$.

Notation like $a b / x y z$ means $(a b) /(x y z)$.

If $\varrho$ is a metric of a set $X$, we let $\varrho(A)$ denote the $\varrho$-diameter of a set $A \subset X$, and $\varrho(A, B)$ is the $\varrho$-distance between nonempty subsets $A, B$ of $X$. In a Banach space $E$ we use the alternative notation $d(x, y)=|x-y|$, and thus $d(A)$ denotes the ordinary diameter of a set $A \subset E$.

I thank the referee for useful comments and corrections. 


\section{Relative, BOUNDED AND INNER UNIFORMITY}

2.1. Summary. An alternative title for this section could be: Handbook on uniform domains and their generalizations. We extend the theory of uniform and inner uniform domains in a Banach space to domains that are uniform relative to a subset or boundedly uniform. The proofs are often rather easy modifications of the absolute (nonrelative) case. In addition to the $\mathrm{QH}$ approach given in the introduction, we define relative and bounded uniformity in terms of cigars. In 2.42 we give a new characterization for uniform domains in terms of neargeodesics. This is applied in 2.44 to show that uniform domains are subinvariant under QH maps.

2.2. Terminology. We recall some concepts introduced in [Vä6]. Let $h \geq 0$ and let $\gamma$ be an arc in a metric space $(X, \varrho)$. The $h$-coarse length of $\gamma$ is defined as

$$
l(\gamma, h)=\sup \sum_{j=1}^{k} \varrho\left(x_{j-1}, x_{j}\right),
$$

where the supremum is taken over all finite sequences $\left(x_{0}, \ldots, x_{k}\right)$ of successive points of $\gamma$ such that $\varrho\left(x_{j-1}, x_{j}\right) \geq h$ for all $1 \leq j \leq k$. If there is no such sequence, then $l(\gamma, h)=0$. For $h=0$ we obtain the ordinary length $l(\gamma)=l(\gamma, 0)$ of $\gamma$. If $\gamma$ is compact and $h>0$, then $l(\gamma, h)<\infty$. The basic theory of coarse length is given in [Vä6, Sec. 4].

We shall use the coarse length in the case where $X=G \varsubsetneqq E$ is a domain and $\varrho=k$ is the $\mathrm{QH}$ metric of $G$, defined in 1.1. We let $l_{k}(\gamma, h)$ denote the $h$-coarse $\mathrm{QH}$ length of an arc $\gamma \subset G$. Then $l_{k}(\gamma, 0)=l_{k}(\gamma)$ is the QH length of $\gamma$, defined by $(1.2)$.

A metric space $(X, \varrho)$ is c-quasiconvex if each pair of points $a, b \in X$ can be joined by an arc $\gamma \subset X$ with $l(\gamma) \leq c \varrho(a, b)$. In particular, an arc $\gamma \subset X$ is $c$-quasiconvex if and only if $l(\gamma[x, y]) \leq c \varrho(x, y)$ for all $x, y \in \gamma$.

An arc $\gamma$ in a domain $G$ is a $c$-neargeodesic if $\gamma$ is $c$-quasiconvex in the $\mathrm{QH}$ metric. In other words,

$$
l_{k}(\gamma[x, y]) \leq c k(x, y)
$$

for all $x, y \in \gamma$. Given points $a, b \in G$ and a number $c>1$, we can always join $a$ and $b$ by a $c$-neargeodesic in $G$; see [Vä6, 3.3]. If $\operatorname{dim} E<\infty$, this is possible with $c=1$; then $\gamma$ is a geodesic of the $\mathrm{QH}$ metric.

More generally, let $h \geq 0$ and $c \geq 1$. An arc $\gamma \subset G$ is $(c, h)$-solid in $G$ if

$$
l_{k}(\gamma[x, y], h) \leq c k(x, y)
$$

for all $x, y \in \gamma$. Thus $(c, 0)$-solid arcs are $c$-neargeodesics. Alternatively, the solid arcs can be characterized as coarsely bilipschitz images of real intervals. We prove this in 2.7 , but the result is not needed in this paper.

Let $e$ be a metric of $G$ satisfying (1.8). Recall from 1.12 that $e(A)$ is the $e$ diameter of a set $A \subset G$. If $e \leq \varrho_{G}$ and $A$ is connected, then $e(A)=d(A)$ is independent of $e$ by $[\mathrm{V} \ddot{4} 4,2.13]$, but $\lambda(A)$ can be larger. On the other hand, the length of an arc $\gamma \subset G$ in each metric $e$ is easily seen to be equal to the ordinary length $l(\gamma)$.

The following two results are $\lambda$-versions of [Vä5, 2.2(1)] and [Vä6, 4.5]. In fact, the proofs of the original results also give these stronger versions. Of course, they are true if $\lambda$ is replaced by any metric $e \leq \lambda$. The first one is well known. 
2.5. Lemma. For all $a, b \in G$ we have

$$
\begin{aligned}
k(a, b) & \geq j_{G}(a, b ; \lambda)=\log \left(1+\frac{\lambda_{G}(a, b)}{\delta(a) \wedge \delta(b)}\right), \\
\lambda_{G}(a, b) & \leq(\delta(a) \wedge \delta(b))\left(e^{k(a, b)}-1\right) .
\end{aligned}
$$

2.6. Lemma. Suppose that $r>0$ and that $\gamma$ is a $(c, h)$-solid arc in $G \cap(\partial G+\bar{B}(r))$. Then

$$
\lambda_{G}(\gamma) \leq M r\left(h \vee l_{k}(\gamma, h)\right),
$$

where $M=M(h)=\left(e^{h}-1\right) / h$ for $h>0$ and $M(0)=1$. If $h=0$, that is, if $\gamma$ is a c-neargeodesic, then

$$
l(\gamma) \leq r l_{k}(\gamma)
$$

2.7. Proposition. Let $J$ be a real interval, and let $g: J \rightarrow G$ be an embedding such that

$$
(|x-y|-C) / M \leq k(g x, g y) \leq M|x-y|+C
$$

for all $x, y \in J$. Then $\gamma=g J$ is a $(c, h)$-solid arc in $G$ with $c=2 M(M+1)$, $h=(2 M+1) C$. Conversely, if $\gamma \subset G$ is a $(c, h)$-solid arc, there is an interval $J \subset \mathrm{R}$ and a homeomorphism $g: J \rightarrow \gamma$ such that (2.8) holds with $M=c, C=2 h$.

Proof. The first part of the proposition follows directly from [Vä6, 4.11]. We prove the second part in the case where $\gamma$ has precisely one endpoint $a$; the other cases are obtained by easy modifications.

Choose inductively successive points $x_{0}, x_{1}, \ldots$ of $\gamma$ such that $x_{0}=a$ and $x_{j}$ is the first point after $x_{j-1}$ with $k\left(x_{j-1}, x_{j}\right)=h$. If no such point exists for $j=N$, the process stops, and we get a finite sequence $x_{0}, \ldots, x_{N-1}$. In this case we set $J=[0, N h)$, otherwise $J=[0, \infty)$.

Set $\Delta_{j}=[(j-1) h, j h)$, and choose homeomorphisms $g_{j}: \Delta_{j} \rightarrow \gamma\left[x_{j-1}, x_{j}\right)$ with $g_{j}((j-1) h)=x_{j-1}$. In the finite case, we also choose a homeomorphism $g_{N}: \Delta_{N} \rightarrow \gamma \backslash \gamma\left[a, x_{N-1}\right)$. We show that the map $g: J \rightarrow \gamma$, defined by $g \mid \Delta_{j}=g_{j}$, satisfies (2.8).

Suppose that $x \in \Delta_{j}$ and $y \in \Delta_{k}$ with $k=j+s \geq j$. If $s \leq 1$, then $k(g x, g y) \leq$ $2 h$. Assume that $s \geq 2$. Now $(s-1) h \leq|x-y| \leq(s+1) h$, and

$$
k(g x, g y) \leq 2 h+\sum_{i=j+1}^{k-1}\left|x_{i-1}-x_{i}\right|=(s+1) h \leq|x-y|+2 h,
$$

which implies the second inequality of (2.8). Since $k\left(x_{i-1}, x_{i}\right)=h$ for all $i$, we have $l_{k}(\gamma[g x, g y], h) \geq(s-1) h$. By the $(c, h)$-solidity of $\gamma$, this implies that $(s-1) h \leq$ $c k(g x, g y)$, and we obtain the first inequality of (2.8) with $M=c, C=2 h$.

2.9. Quasihyperbolic $\psi$-uniformity. Let $\psi:[0, \infty) \rightarrow[0, \infty)$ be a homeomorphism. A domain $G$ is $\mathrm{QH} \psi$-uniform if

$$
k_{G}(a, b) \leq \psi\left(r_{G}(a, b)\right)
$$

for all $a, b \in G$. For notation, see (1.3) and (1.4). In the special case $\psi(t)=$ $c \log (1+t)$, we obtain the QH $c$-uniform domains defined in 1.1. The terminology is somewhat ambiguous, but we shall always use $\psi$ for functions and $c$ for constants. 
For example, every convex domain is $\mathrm{QH} \psi$-uniform with $\psi(t)=t$. However, to get interesting results we must assume that

$$
\lim _{t \rightarrow \infty} \frac{\psi(t)}{t}=0 .
$$

Functions $\psi$ with this property are called slow. For example, the function $\psi(t)=$ $c \log (1+t)$ is slow. In fact, if $G$ is $\mathrm{QH} \psi$-uniform with a slow $\psi$, then $G$ is $\mathrm{QH}$ $c$-uniform with a constant $c=c(\psi)[\mathrm{Vä6}, 6.16]$.

If $A \subset G$ and if (2.10) holds for all $a, b \in A$, we say that $G$ is QH $\psi$-uniform relative to $A$, or briefly QH $\psi$-uniform rel $A$. If $R>0$ and if (2.10) holds for all $a, b \in G$ with $|a-b| \leq R$, then $G$ is said to be $R$-boundedly $\mathrm{QH} \psi$-uniform. For $\psi(t)=c \log (1+t)$, we obtain the corresponding $\mathrm{QH} c$-uniform properties defined in 1.1 .

Inner and $e$-versions of $\mathrm{QH} \psi$-uniformity are defined in the obvious way. For example, $G$ is $\mathrm{QH}(\psi, e)$-uniform if

$$
k_{G}(a, b) \leq \psi\left(r_{G}(a, b ; e)\right)
$$

for all $a, b \in G$, where $r_{G}(a, b ; e)$ is defined in (1.10). Furthermore, $G$ is $R$-boundedly $\mathrm{QH}(\psi, e)$-uniform if this is true whenever $e(a, b) \leq R$. For $e=\lambda$ we obtain the properties QH inner $\psi$-uniform, QH inner $\psi$-uniform rel $A$, and $R$-boundedly $\mathrm{QH}$ inner $\psi$-uniform.

Given a slow function $\psi$ and numbers $c \geq 1, h \geq 0$, we let $M_{1}(c, h, \psi)$ denote the smallest number $M_{1} \geq 1$ such that

$$
\left\{\begin{array}{l}
t>M(h) c \psi(2 t), \\
\psi^{-1}(h / c) \leq 2 M_{1},
\end{array} \text { if } t>M_{1},\right.
$$

where $M(h)=\left(e^{h}-1\right) / h$ is the number of 2.6 .

2.12. Solid arcs in uniform domains. Solid arcs and neargeodesics play an important role in the free quasiworld, since the solidity property of arcs is preserved by freely quasiconformal maps and, more generally, by solid and coarsely QH maps. It is therefore useful to have some knowledge of the geometric properties of these arcs. For uniform domains, such properties were established in [Vä6, Sec. 6]. Our next goal is to obtain relative, bounded, and $e$-versions of these results.

We start with two central lemmas. Roughly speaking, the first result says that a solid arc must escape the boundary of the domain; it cannot travel a long distance near the boundary. In the second lemma we show that if a solid arc dives from a distance $\delta\left(x_{0}\right)$ to the distance $\delta(x)=q \delta\left(x_{0}\right)$ with sufficiently small $q$, then the life of $\gamma$ after the point $x$ is fairly short.

The proofs of both lemmas follow the proofs of the original results [Vä6, 6.10, 6.11]. Since QH $\psi$-uniform implies QH inner $\psi$-uniform and since $d(\gamma) \leq \lambda(\gamma)$, they are stronger than the original versions.

2.13. Escape lemma. Suppose that $\psi$ is slow, that $G$ is $\mathrm{QH}$ inner $\psi$-uniform rel $A$, and that $\gamma \subset A \cap(\partial G+\bar{B}(r))$ is a $(c, h)$-solid arc in $G$. Then

$$
\lambda_{G}(\gamma) \leq 4 M_{1} r
$$

where $M_{1}=M_{1}(c, h, \psi)$ is defined by (2.11). This is also true if $G$ is $R$-boundedly $\mathrm{QH}$ inner $\psi$-uniform and if $\gamma \subset G \cap\left(\partial G+\bar{B}\left(R / 4 M_{1}\right)\right)$. In this case we have $\lambda_{G}(\gamma) \leq R$.

If $h=0$, then (2.14) is replaced by $l(\gamma) \leq 4 M_{1} r$. 
Proof. Assume first that $h>0$. We may assume that $\gamma$ is a compact arc with endpoints $a$ and $b$. We first show that if $\delta(a) \wedge \delta(b) \geq r / 2$, then $\lambda(\gamma) \leq M_{1} r$. Setting $t=\lambda(\gamma) / r$ we get

$$
l_{k}(\gamma, h) \leq c k(a, b) \leq c \psi\left(r_{G}(a, b ; \lambda)\right) \leq c \psi(2 t) .
$$

If $c \psi(2 t) \leq h$, then $2 t \leq \psi^{-1}(h / c) \leq 2 M_{1}$ by $(2.11)$. If $c \psi(2 t) \geq h$, Lemma 2.6 gives

$$
t r=\lambda(\gamma) \leq M r(h \vee c \psi(2 t))=\operatorname{Mrc\psi }(2 t) .
$$

By (2.11) this implies that $t \leq M_{1}$.

In the general case we choose $a_{0} \in \gamma$ such that $\delta\left(a_{0}\right)$ is maximal. We may assume that $\delta\left(a_{0}\right)=r$. Considering $a_{0}$ as the first point of the $\operatorname{arc} \gamma^{\prime}=\gamma\left[a_{0}, a\right]$ we divide $\gamma^{\prime}$ into subarcs $\gamma_{j}=\gamma\left[a_{j-1}, a_{j}\right], 1 \leq j \leq N$, where $a_{j}$ is the last point of $\gamma^{\prime}$ with $\delta\left(a_{j}\right)=2^{-j} r$, and $a_{N}=a$. By the special case considered above we have $\lambda\left(\gamma_{j}\right) \leq 2^{-j+1} M_{1} r$ for all $j$, and hence $\lambda\left(\gamma^{\prime}\right)<2 M_{1} r$. Similarly $\lambda\left(\gamma\left[a_{0}, b\right]\right)<2 M_{1} r$, and (2.14) follows.

Observe that if $\gamma$ is a compact arc, then (2.14) holds as a strict inequality.

Suppose that $G$ is $R$-boundedly $\mathrm{QH}$ inner $\psi$-uniform and that $\gamma \subset G \cap$ $\left(\partial G+\bar{B}\left(R / 4 M_{1}\right)\right)$. If $\lambda(\gamma) \leq R$, then $\gamma$ is $\mathrm{QH}$ inner $\psi$-uniform rel $\gamma$, and (2.14) follows by the first part of the lemma. If $\lambda(\gamma)>R$, there is a compact subarc $\beta \subset \gamma$ with $\lambda(\beta)=R$. Since $\beta$ is $(c, h)$-solid, the first part of the lemma gives the contradiction $R=\lambda(\beta)<4 M_{1} R / 4 M_{1}=R$.

The proof for the case $h=0$ is similar but easier.

2.15. Diving lemma. Suppose that $\psi$ is slow and that $c \geq 1, h \geq 0$. Then there is $q=q(c, h, \psi) \in(0,1)$ with the following property:

Let $G$ be a $\mathrm{QH}$ inner $\psi$-uniform domain rel $A$, and let the arc $\gamma \subset A$ be $(c, h)$ solid in $G$ with endpoints $a_{0}$ and $a_{1}$. If $x \in \gamma$ and $\delta(x) \leq q \delta\left(a_{0}\right)$, then for $\gamma_{x}=$ $\gamma\left[x, a_{1}\right]$ we have

$$
\lambda_{G}\left(\gamma_{x}\right) \leq 4 M_{1} \delta(x) / q
$$

where $M_{1}=M_{1}(c, h, \psi)$ is defined by (2.11). This is also true if $G$ is $R$-boundedly QH inner $\psi$-uniform and $\delta\left(a_{0}\right) \leq R / 4 M_{1}$.

If $h=0$, then $l\left(\gamma_{x}\right) \leq 4 M_{1} \delta(x) / q$.

Proof. Writing $K=2\left(h \vee c \psi\left(4 M_{1}\right)\right)$, we show that the lemma holds with $q=e^{-K}$.

In the situation of the lemma we set $r=\delta(x) / q$. It suffices to show that $\gamma_{x} \subset$ $\partial G+\bar{B}(r)$, since the result then follows from the Escape lemma 2.13.

Assume that $\gamma_{x} \not \subset \partial G+\bar{B}(r)$. Since $\delta\left(a_{0}\right) \geq r$, there are $x_{1}, x_{2} \in \gamma$ such that $\delta\left(x_{1}\right)=\delta\left(x_{2}\right)=r$ and $x \in \gamma\left[x_{1}, x_{2}\right] \subset \partial G+\bar{B}(r)$. Suppose first that $h>0$. Then $\lambda\left(x_{1}, x_{2}\right) \leq \lambda(\gamma) \leq 4 M_{1} r$ by 2.13. Since $G$ is $\mathrm{QH}$ inner $\psi$-uniform rel $\gamma$ and since $\gamma$ is $(c, h)$-solid, we obtain

$$
l_{k}\left(\gamma\left[x_{1}, x\right], h\right) \leq l_{k}\left(\gamma\left[x_{1}, x_{2}\right], h\right) \leq c k\left(x_{1}, x_{2}\right) \leq c \psi\left(r_{G}\left(x_{1}, x_{2} ; \lambda\right)\right) \leq c \psi\left(4 M_{1}\right) .
$$

By 2.5 this implies that

$$
\log \left(1+\left|x_{1}-x\right| / q r\right) \leq k\left(x_{1}, x\right) \leq h \vee l_{k}\left(\gamma\left[x_{1}, x\right], h\right) \leq K / 2 .
$$

On the other hand, $\left|x_{1}-x\right| \geq \delta\left(x_{1}\right)-\delta(x)=(1-q) r$, and hence $1+\left|x_{1}-x\right| / q r \geq q$. These estimates give the contradiction $K \leq K / 2$.

The proof for the case $h=0$ is similar. 
2.16. Geometric approach to uniform domains. We recall the approach to uniform domains based on cigars. Let $\gamma \subset E$ be an arc with endpoints $a$ and $b$. For $x \in \gamma$ we set

$$
\zeta_{d}(x)=\zeta_{d}(x, \gamma)=d(\gamma[a, x]) \wedge d(\gamma[x, b]) .
$$

If $\gamma$ is rectifiable, we also define the function

$$
\zeta_{l}(x)=\zeta_{l}(x, \gamma)=l(\gamma[a, x]) \wedge l(\gamma[x, b]) .
$$

For $c \geq 1$, the sets

$$
\begin{aligned}
\operatorname{cig}_{d}(\gamma, c) & =\bigcup\left\{B\left(x, \zeta_{d}(x) / c\right): x \in \gamma \backslash\{a, b\}\right\}, \\
\operatorname{cig}_{l}(\gamma, c) & =\bigcup\left\{B\left(x, \zeta_{l}(x) / c\right): x \in \gamma \backslash\{a, b\}\right\}
\end{aligned}
$$

are the diameter c-cigar and the length c-cigar, respectively, with core $\gamma$. The length cigar is only defined for a rectifiable $\gamma$.

Let $G$ be a domain and let $\gamma \subset G$ be a rectifiable arc with endpoints $a$ and $b$. We say that $\gamma$ satisfies the uniformity conditions in $G$ with a constant $c \geq 1$, or briefly, $\gamma$ is $c$-uniform in $G$, if

(1) $\operatorname{cig}_{l}(\gamma, c) \subset G$,

(2) $l(\gamma) \leq c|a-b|$.

Condition (1) is the cigar condition, and (2) is the turning condition. Alternatively, (1) can be written as

$\left(1^{\prime}\right) \zeta_{l}(x, \gamma) \leq c \delta(x)$

for all $x \in \gamma$.

A domain $G$ is said to be a $c$-uniform domain if each pair $a, b \in G$ can be joined by a rectifiable arc $\gamma$ satisfying the $c$-uniformity conditions (1) and (2). For a set $A \subset G$, we say that $G$ is c-uniform rel $A$ if each pair $a, b \in A$ has this property. If $R>0$ and if each pair $a, b \in G$ with $|a-b| \leq R$ has this property, then $G$ is said to be $R$-boundedly c-uniform.

The $(\varepsilon, \delta)$ domains considered by Jones [Jo2] are closely related to the $R$-boundedly $c$-uniform domains. See 3.14 .

The $e$-versions of these properties are obtained by replacing (2) by the condition (2e) $l(\gamma) \leq c e(a, b)$.

A rectifiable arc $\gamma \subset G$ with endpoints $a, b$ is said to be $(c, e)$-uniform in $G$ if it satisfies (1) and (2e). If $e=\lambda$, then $\gamma$ is inner c-uniform in $G$. The domain $G$ is $(c, e)$-uniform if each pair $a, b \in G$ can be joined by a $(c, e)$-uniform arc. The properties $(c, e)$-uniform rel $A, R$-boundedly $(c, e)$-uniform, inner $c$-uniform, inner $c$-uniform rel $A$, and $R$-boundedly inner $c$-uniform are defined in the obvious way. For example, $G$ is $R$-boundedly $(c, e)$-uniform if each pair $a, b$ with $e(a, b) \leq R$ can be joined by a $(c, e)$-uniform arc.

The extreme cases $e=d \mid G$ and $e=\lambda_{G}$ are the most interesting. The intermediate case $e=\varrho_{G}$, defined by (1.11), appears in the literature but usually together with a modified cigar condition (1), where $\operatorname{cig}_{l}$ is replaced by $\operatorname{cig}_{d}$ or by the distance cigar defined by the function $\zeta(x, \gamma)=|x-a| \wedge|x-b|$. If $\operatorname{dim} E<\infty$, these variations yield the same class of domains as $e=\lambda$, but this is not true in arbitrary Banach spaces. These questions will be considered in Section 3.

The following result follows immediately from the definitions: 
2.17. Theorem. A c-uniform domain is c-quasiconvex and inner c-uniform. Conversely, if $G$ is $c_{0}$-quasiconvex and inner c-uniform, then $G$ is cc $c_{0}$-uniform.

2.18. Examples. 1. Recall that $G$ is a $c$-John domain if each pair $a, b \in G$ can be joined by an $\operatorname{arc} \gamma$ satisfying the cigar condition (1) of 2.16. Hence an inner $c$ uniform domain is $c$-John. If $C$ is any compact subset of the line segment $\left[0, e_{1}\right] \subset$ $\mathrm{R}^{2}$, then $B^{2} \backslash C$ is $c$-John with a universal $c$, but it need not be inner uniform. This example is due to J. Heinonen.

2. On the other hand, every simply connected $c$-John domain in $R^{2}$ is inner $c_{1}$-uniform with $c_{1}=c_{1}(c)$; cf. [BV1, p. 43].

3. More examples of inner uniform domains can be constructed by locally bilipschitz maps; see Theorem 2.21.

4. Let $F$ be the ray $\left\{t e_{1}: t \geq 0\right\} \subset \mathrm{R}^{2}$. The domain $\mathrm{R}^{2} \backslash F$ is inner uniform but not uniform. Let $D$ be the parallel strip $\{(x, y):|y|<1\}$. Then $D \backslash F$ is not inner uniform but it is $R$-boundedly inner $c_{R}$-uniform with some constant $c_{R}$ for each $R>0$.

5. It is well known that bounded convex domains are uniform. We shall later make use of the following explicit version of this result.

2.19. Theorem. Suppose that $G$ is a convex domain and that $B\left(x_{0}, R_{1}\right) \subset G \subset$ $B\left(x_{0}, R_{2}\right)$. Then $G$ is c-uniform with $c=2 R_{2} / R_{1}$.

Proof. We first show that the function $\delta: G \rightarrow \mathrm{R}$ is concave, that is,

$$
\delta((1-t) a+t b) \geq(1-t) \delta(a)+t \delta(b)
$$

for all $a, b \in G$ and $0 \leq t \leq 1$. Write $x=(1-t) a+t b, R=(1-t) \delta(a)+t \delta(b)$. Let $w \in E$ with $|w|<1$. Then the points $a^{\prime}=a+\delta(a) w$ and $b^{\prime}=b+\delta(b) w$ lie in $G$. By convexity, $z=(1-t) a^{\prime}+t b^{\prime} \in G$. Since $z=x+R w$, we obtain $x+B(R) \subset G$, and (2.20) follows.

We may assume that $x_{0}=0$. Let $a, b \in G, a \neq b$. Set $r=|a-b| / 2$ and $z=(a+b) / 2$. We consider two cases.

Case 1. $|z| \geq r$. Set $y=z-r z /|z|$. We show that the $\operatorname{arc} \gamma=[a, y] \cup[y, b]$ has the uniformity properties. Since

$$
l(\gamma)=|a-y|+|y-b| \leq|a-z|+|z-y|+|y-z|+|z-b|=4 r=2|a-b|,
$$

the turning condition holds with the constant 2 .

To prove the cigar condition it suffices to show that $|x-a| \leq c \delta(x)$ for $x \in[a, y]$. Write $x=(1-t) a+t y, 0 \leq t \leq 1$. By the concavity of $\delta$ we get

$$
\begin{aligned}
\delta(x) & \geq(1-t) \delta(a)+t \delta(y) \\
& \geq t \delta(y) \geq t(1-r /|z|) \delta(z)+\operatorname{tr} \delta(0) /|z| \geq \operatorname{tr} R_{1} / R_{2}=2 t r / c .
\end{aligned}
$$

Hence

$$
|x-a|=t|a-y| \leq 2 \operatorname{tr} \leq c \delta(x) .
$$

Case 2. $|z| \leq r$. Now we set $\gamma=[a, 0] \cup[0, b]$. Since $|a| \leq|a-z|+|z| \leq 2 r$ and $|b| \leq 2 r$, we again get $l(\gamma) \leq 2|a-b|$. If $x=(1-t) a \in[a, 0]$, then

$$
\delta(x) \geq(1-t) \delta(a)+t \delta(0) \geq t R_{1}=2 R_{2} t / c,
$$

and we again obtain

$$
|x-a|=t|a| \leq 2 r t \leq 2 R_{2} t \leq c \delta(x) .
$$


2.21. Theorem. Suppose that $f: G \rightarrow G^{\prime}$ is a homeomorphism between domains $G \subset E$ and $G^{\prime} \subset E^{\prime}$, and that $f$ is locally $M$-bilipschitz. If $G$ is inner c-uniform, then $G^{\prime}$ is inner $M^{2}$ c-uniform.

Proof. For each rectifiable arc $\alpha \subset G$, we have $l(\alpha) / M \leq l(f \alpha) \leq M l(\alpha)$. Since $f$ and $f^{-1}$ are $M$-Lipschitz on every line segment in the domains and since the spaces are complete, we have $d(x, \partial G) / M \leq d\left(f x, \partial G^{\prime}\right) \leq M d(x, \partial G)$ for all $x \in G$; cf. [Vä5, 4.8]. The theorem follows now easily.

2.22. Comparison of the $\mathbf{Q H}$ and the cigar approach. We want to compare the concepts defined in 2.16 with the QH uniformity properties introduced in 1.1 and in 2.10. It is well known that the properties " $c$-uniform" and "QH $c$-uniform" are quantitatively equivalent for a domain $G$. This was proved by F.W. Gehring and B.G. Osgood in [GO]; a free version appears in [Vä6, 6.16]. Moreover, these properties are quantitatively equivalent to $\mathrm{QH} \psi$-uniformity with a slow $\psi$.

We want to prove relative and bounded $e$-versions of these results. In 2.23 we show that if $G$ is a domain and if $\gamma \subset G$ is a compact $(c, e)$-uniform arc, then the endpoints $a$ and $b$ of $\gamma$ satisfy the QH uniformity condition

$$
k(a, b) \leq 7 c^{3} j_{G}(a, b ; e) .
$$

Hence each $(c, e)$-uniformity property in the cigar sense implies the corresponding QH $(c, e)$-uniformity property with $c \mapsto 7 c^{3}$.

In the converse direction, the situation is more complicated. We show in 2.31 that if $\gamma \subset G$ is a $c$-neargeodesic with endpoints $a, b$, and if $k(a, b) \leq C$, then $\gamma$ satisfies the uniformity conditions with a constant $c_{1}=c_{1}(c, C)$. From an example in 2.28 we see that $c$ cannot be chosen to be independent of $C$. On the other hand, it is well known ([GO],[Vä6]) that if $G$ is $\mathrm{QH} c$-uniform, then each compact $c_{0}$-neargeodesic satisfies the $c_{1}$-uniformity conditions with $c_{1}=c_{1}\left(c, c_{0}\right)$. We show that the corresponding result holds for $(c, e)$-uniformity rel $A$, assuming that $A$ satisfies a $\mathrm{QH}$ quasiconvexity condition. Moreover, an $R$-bounded version is true if $R$ is allowed to change.

2.23. Theorem. Suppose that $\gamma$ is a $(c, e)$-uniform arc in $G$ with endpoints $a, b$. Then

$$
k(a, b) \leq l_{k}(\gamma) \leq c_{1} j_{G}(a, b ; e) \leq c_{1} k(a, b)
$$

with $c_{1}=c_{1}(c) \leq 7 c^{3}$.

Proof. The first inequality is trivial, and the last one follows from 2.5. It remains to prove the middle inequality. Write $\delta=\delta(a) \wedge \delta(b)$ and $r=r_{G}(a, b ; e)=e(a, b) / \delta$. We consider two cases.

Case 1. $r \leq 1 / 2 c$. For every $x \in \gamma$ we have

$$
|x-a| \leq l(\gamma) \leq c e(a, b)=c \delta r \leq \delta(a) / 2,
$$

and hence $\delta(x) \geq \delta(a) / 2$. Consequently,

$$
l_{k}(\gamma) \leq 2 l(\gamma) / \delta(a) \leq 2 c e(a, b) / \delta(a) \leq 2 c r .
$$

Moreover, since $r \leq 1 / 2 c \leq 1 / 2<1$, we obtain $r \log 2 \leq \log (1+r)=j_{G}(a, b ; e)$, and hence (2.24) holds with $c_{1}=2 c / \log 2<3 c$. 
Case 2. $r>1 / 2 c$. Write $L=l(\gamma)$, and let $x_{0} \in \gamma$ be the point with $l\left(\gamma\left[a, x_{0}\right]\right)=$ $L / 2$. Since $L \geq \lambda(a, b) \geq e(a, b)=\delta r>\delta / 2 c$, there is $a_{1} \in \gamma\left[a, x_{0}\right]$ with $l\left(\gamma\left[a, a_{1}\right]\right)=$ $\delta / 4 c$. Since $\gamma\left[a, a_{1}\right] \subset \bar{B}(a, \delta(a) / 4)$, we can argue as in Case 1 to obtain

$$
l_{k}\left(\gamma\left[a, a_{1}\right]\right) \leq \frac{2 l\left(\gamma\left[a, a_{1}\right]\right)}{\delta(a)}=\frac{\delta}{2 c \delta(a)} \leq \frac{1}{2 c} \leq \frac{1}{2} .
$$

For the $\operatorname{arc} \beta=\gamma\left[a_{1}, x_{0}\right]$, the cigar condition implies that

$$
l_{k}(\beta) \leq c \int_{\beta} \frac{|d x|}{l(\gamma[a, x])}=c \int_{\delta / 4 c}^{L / 2} \frac{d s}{s}=c \log \frac{2 c L}{\delta} .
$$

Considering similarly the second half $\gamma\left[x_{0}, b\right]$ of $\gamma$ we obtain the estimate $l_{k}(\gamma) \leq$ $1+2 c \log (2 c L / \delta)$. By the turning condition and by the inequality $\log (M r) \leq$ $M \log (1+r)$, valid for $M \geq 1$, this yields

$$
l_{k}(\gamma) \leq 1+2 c \log \left(2 c^{2} r\right) \leq 1+4 c^{3} j_{G}(a, b ; e) .
$$

Since $r>1 / 2 c$, we have

$$
j_{G}(a, b ; e)>\log (1+1 / 2 c)>1 / 3 c .
$$

These estimates give (2.24) with $c_{1}=3 c+4 c^{3} \leq 7 c^{3}$.

2.25. Theorem. Suppose that $\gamma \subset G$ is a rectifiable compact arc, that $\operatorname{cig}_{l}(\gamma, c) \subset$ $G$ and that $\gamma$ is c-quasiconvex in the inner metric $\lambda_{G}$. Then $\gamma$ is a $c_{1}$-neargeodesic in $G$ with $c_{1}=c_{1}(c) \leq 7 c^{3}$.

Proof. Since each subarc of $\gamma$ is $c$-uniform in $G$, the theorem follows from 2.23.

2.26. Remark. It follows from 2.25 that if $\operatorname{cig}_{l}(\gamma, c) \subset G$ and if $\gamma$ is $c$-quasiconvex in the norm metric, then $\gamma$ is a $7 c^{3}$-neargeodesic in $G$.

2.27. Theorem. If $G$ is $(c, e)$-uniform rel $A$, then $G$ is $\mathrm{QH}\left(7 c^{3}, e\right)$-uniform rel $A$. If $G$ is $R$-boundedly c-uniform, then $G$ is $R$-boundedly $\mathrm{QH}\left(7 c^{3}, e\right)$-uniform.

Proof. This is a corollary of 2.23 .

2.28. Example. It is natural to ask whether the inequality $k(a, b) \leq c j_{G}(a, b)$ for a pair of points $a, b$ implies that these points can be joined by a $c_{1}$-uniform arc in $G$ with $c_{1}=c_{1}(c)$. The following example shows that the answer is negative.

Let $G \subset \mathrm{R}^{2}$ be the domain $\left\{x: x_{1}<0\right.$ or $\left.1<\left|x_{2}\right|<3\right\}$. Let $c \geq 1$, and set $t=e^{-10 c}, a=(2 c, 1+t), b=(2 c,-1-t)$. Then $j_{G}(a, b)=\log (1+|a-b| / t)>$ $\log (1 / t)=10 c$. Let $\beta$ be the broken line with vertices $a,(2 c, 2),(-1,2),(-1,-2)$, $(2 c,-2), b$. Then

$$
k(a, b) \leq l_{k}(\beta) \leq 2 \log (1 / t)+4 c+6 \leq 30 c<3 j_{G}(a, b) .
$$

In other words, $G$ is QH 3-uniform rel $\{a, b\}$. However, it is easy to see that if $\gamma \subset G$ is an arc joining $a$ and $b$, then $\gamma$ satisfies neither of the $c$-uniformity conditions.

We give several results which show that with additional hypotheses, a $\mathrm{QH}$ uniformity condition like $k(a, b) \leq c j_{G}(a, b)$ implies that a neargeodesic with endpoints $a, b$ satisfies the uniformity conditions.

2.29. Cigar theorem. Suppose that $G$ is $\mathrm{QH}(\psi, e)$-uniform rel $A$ with a slow $\psi$ and that $\gamma \subset A$ is a compact c-neargeodesic in $G$. Then $\gamma$ is $\left(c_{1}, e\right)$-uniform in $G$ with $c_{1}=c_{1}(c, \psi)$. This is also true if $G$ is $R$-boundedly $\mathrm{QH}(\psi, e)$-uniform and $\gamma \subset G \cap\left(\partial G+\bar{B}\left(R / 4 M_{1}\right)\right)$, where $M_{1}=M_{1}(c, 0, \psi)$ is defined by (2.11). 
Proof. Let $a_{0}$ and $a_{1}$ be the endpoints of $\gamma$, and let $x_{0} \in \gamma$ be a point with maximal $\delta\left(x_{0}\right)$. Let $q=q(c, 0, \psi)$ be the number given by the Diving lemma 2.15. If $x \in \gamma\left[a_{0}, x_{0}\right]$ and $\delta(x) \leq q \delta\left(x_{0}\right)$, then 2.15 implies that

$$
l\left(\gamma\left[a_{0}, x\right]\right) \leq 4 M_{1} \delta(x) / q .
$$

If $x \in \gamma\left[a_{0}, x_{0}\right]$ and $\delta(x) \geq q \delta\left(x_{0}\right)$, then the Escape lemma 2.13 with $r=\delta\left(x_{0}\right)$ gives

$$
l\left(\gamma\left[a_{0}, x\right]\right) \leq 4 M_{1} \delta\left(x_{0}\right) \leq 4 M_{1} \delta(x) / q .
$$

Considering similarly the arc $\gamma\left[a_{1}, x_{0}\right]$ we see that $\operatorname{cig}_{l}\left(\gamma, c_{1}\right) \subset G$ with $c_{1}=$ $c_{1}(c, \psi)=4 M_{1} / q$.

To prove the turning condition we may assume that $\delta\left(a_{0}\right) \leq \delta\left(a_{1}\right)$. Set $t=$ $e\left(a_{0}, a_{1}\right)$ and $r=\delta\left(a_{0}\right)$. We must show that $l(\gamma) \leq c_{2} t$ with $c_{2}=c_{2}(c, \psi)$. We consider two cases.

Case 1. $r \leq t$. We may assume that $l(\gamma)>2 t$. Choose points $b_{0}, b_{1} \in \gamma$ such that $l\left(\gamma\left[a_{0}, b_{0}\right]\right)=l\left(\gamma\left[a_{1}, b_{1}\right]\right)=t$. Then $e\left(a_{0}, b_{0}\right) \leq \lambda\left(a_{0}, b_{0}\right) \leq t$ and similarly $e\left(a_{1}, b_{1}\right) \leq t$. Since $\delta\left(b_{j}\right) \geq t / c_{1}$ by the cigar condition, we obtain

$$
e\left(b_{0}, b_{1}\right) \leq e\left(b_{0}, a_{0}\right)+e\left(a_{0}, a_{1}\right)+e\left(a_{1}, b_{1}\right) \leq 3 t \leq 3 c_{1}\left(\delta\left(b_{0}\right) \wedge \delta\left(b_{1}\right)\right) .
$$

Since $G$ is $\mathrm{QH}(\psi, e)$-uniform rel $\gamma$, this implies that

$$
k\left(b_{0}, b_{1}\right) \leq \psi\left(r_{G}\left(b_{0}, b_{1} ; e\right)\right) \leq \psi\left(3 c_{1}\right) .
$$

For each $x \in \gamma\left[b_{0}, b_{1}\right]$ this yields

$$
k\left(x, b_{0}\right) \leq l_{k}\left(\gamma\left[b_{0}, b_{1}\right]\right) \leq c k\left(b_{0}, b_{1}\right) \leq c \psi\left(3 c_{1}\right) .
$$

By [Vä5, 2.2(1)] or by 2.5 we further obtain $\left|x-b_{0}\right| \leq \delta\left(b_{0}\right)\left(e^{c \psi\left(3 c_{1}\right)}-1\right)$. Since $\delta\left(b_{0}\right) \leq \delta\left(a_{0}\right)+\left|a_{0}-b_{0}\right| \leq r+t \leq 2 t$, this gives

$$
\delta(x) \leq \delta\left(b_{0}\right)+\left|x-b_{0}\right| \leq \delta\left(b_{0}\right) e^{c \psi\left(3 c_{1}\right)} \leq c_{3} t
$$

with $c_{3}=c_{3}(c, \psi)$. Hence

$$
l\left(\gamma\left[b_{0}, b_{1}\right]\right) \leq c_{3} t l_{k}\left(\gamma\left[b_{0}, b_{1}\right]\right) \leq c c_{3} t k\left(b_{0}, b_{1}\right) .
$$

This and (2.30) give the turning condition $l(\gamma) \leq c_{2} t$ with $c_{2}=2+c c_{3} \psi\left(3 c_{1}\right)$.

Case 2. $r>t$. This case is independent of the uniformity properties of $G$. The proof of [Vä6, 6.12] gives $l(\gamma) \leq c_{2}\left|a_{0}-a_{1}\right|$ with $c_{2}=\max \left\{u e^{2 c / u}-1: u \geq 1\right\}$.

2.31. Lemma. Let $\gamma$ be a c-neargeodesic in $G$ with endpoints $a, b$ such that $k(a, b) \leq$ $C$. Then $\gamma$ is $c_{1}$-uniform in $G$ with $c_{1}=c_{1}(c, C)$.

Proof. Set $r=\delta(a)$ and $B=B(a, r / 2)$. We consider two cases.

Case 1. $\gamma \subset B$. Since $r / 2 \leq \delta(x) \leq 2 r$ for all $x \in B$, we have by [Vä5, 2.5]

$$
l(\gamma) \leq 2 r l_{k}(\gamma) \leq 2 \operatorname{rck}(a, b) \leq 4 c|a-b|
$$

which is the turning condition. For all $x \in \gamma$, this implies the cigar condition

$$
\zeta_{l}(x, \gamma) \leq l(\gamma) / 2 \leq 2 c|a-b| \leq c r \leq 2 c \delta(x) .
$$


Case 2. $\gamma \not \subset B$. Choose a point $y \in \gamma \backslash B$ and set $t=|a-b|$. By (1.5) we have $k(a, y) \geq j_{G}(a, y) \geq \log \frac{3}{2}>1 / 3$, and hence

$$
1 / 3<l_{k}(\gamma) \leq c k(a, b)
$$

If $t \leq r / 2$, then [Vä5, 2.5] gives $k(a, b) \leq 2 t / r$, and hence

$$
r \leq 6 c t,
$$

which is trivially true also in the case $t \geq r / 2$.

Set $L=l(\gamma)$, and let $\gamma^{0}:[0, L] \rightarrow \gamma$ be the arclength parametrization with $\gamma^{0}(0)=a$. For each $0 \leq s \leq L$ we have

$$
\delta\left(\gamma^{0}(s)\right) \leq \delta(a)+\left|a-\gamma^{0}(s)\right| \leq r+s,
$$

and hence

$$
l_{k}(\gamma) \geq \int_{0}^{L} \frac{d s}{r+s}>\log \frac{L}{r} .
$$

Since $l_{k}(\gamma) \leq c k(a, b) \leq c C$, this and (2.32) imply the turning condition $L \leq 6 c e^{c C} t$.

For each $x \in \gamma$ we have by [Vä5, 2.2(1)]

$$
\log \frac{r}{\delta(x)} \leq k(a, x) \leq l_{k}(\gamma) \leq c k(a, b) \leq c C .
$$

Since $t \leq r e^{k(a, b)} \leq r e^{C}$, we obtain the cigar condition

$$
\zeta_{l}(x, \gamma) \leq L / 2 \leq 3 c e^{c C} t \leq 3 c e^{3 c C} \delta(x) .
$$

2.33. Theorem. Suppose that $A \subset G$ and that each pair of points in $A$ can be joined by a $c_{0}$-neargeodesic $\gamma \subset A$ of $G$. Let $d \mid G \leq e \leq \lambda_{G}$. Then the following conditions are quantitatively equivalent:

(1) $G$ is $(c, e)$-uniform rel $A$.

(2) $G$ is QH $(c, e)$-uniform rel $A$.

(3) $k(x, y) \leq c j_{G}(x, y ; e)+c^{\prime}$ for all $x, y \in A$.

(4) $G$ is $\mathrm{QH}(\psi, e)$-uniform rel $A$ with a slow $\psi$.

Proof. By quantitativeness we mean that the quantities of each condition depend only on $c_{0}$ and on the quantities of another condition.

The implications $(3) \Leftarrow(2) \Rightarrow(4)$ are trivial, $(1) \Rightarrow(2)$ holds for all $A \subset G$ by 2.23 , the proof for $(3) \Rightarrow(2)$ is given in [Vä6, 6.15], and $(4) \Rightarrow(1)$ follows from 2.29 .

2.34. Remarks. 1. The case $A=G, e=d \mid G$ of 2.33 was given in [Vä6, 6.16]. The case $A=G, e=\lambda$ gives four quantitatively equivalent conditions for the inner $c$-uniformity of $G$.

A different new characterization of the classical case $e=d \mid G$, based on subinvariance of neargeodesics, will be given in 2.42 .

2. It follows from Theorem 2.33 that one can replace the condition "QH inner $\psi$-uniform" by "inner $c$-uniform" in the case $A=G$ of the Escape lemma 2.13 and of the Diving lemma 2.15. Similarly, one can replace the condition "QH $(\psi, e)$ uniform" in the case $A=G$ of the Cigar theorem 2.29 by "(c,e)-uniform". In the rest of the paper, we often apply these modifications without further notice.

We next turn to the bounded version of 2.33. 
2.35. Lemma. Suppose that $G$ is R-boundedly $\mathrm{QH}(\psi, e)$-uniform with a slow $\psi$. Suppose also that $\gamma$ is a c-neargeodesic in $G$ with endpoints $a_{0}, a_{1}$, and that $e\left(a_{0}, a_{1}\right) \leq R / 25 M_{1}$, where $M_{1}=M_{1}(c, 0, \psi)$ is given by (2.11). Then $\gamma$ is a $\left(c_{1}, e\right)$-uniform arc in $G$ with $c_{1}=c_{1}(c, \psi)$.

Proof. Set $R_{1}=R / 25 M_{1}$ and $t=e\left(a_{0}, a_{1}\right)$. We may assume that $\delta\left(a_{0}\right) \leq \delta\left(a_{1}\right)$. If $\delta\left(a_{1}\right) \geq 2 R_{1}$, then $\left|a_{0}-a_{1}\right| \leq e\left(a_{0}, a_{1}\right) \leq \delta\left(a_{1}\right) / 2$. This implies that $k\left(a_{0}, a_{1}\right) \leq 1$, and the assertion follows from 2.31. Thus we may assume that $\delta\left(a_{1}\right) \leq 2 R_{1}$. Similarly, we may assume that $t \geq \delta\left(a_{1}\right) / 2$, since otherwise we again get $\left|a_{0}-a_{1}\right| \leq$ $\delta\left(a_{1}\right) / 2$.

Orient $\gamma$ so that $a_{0}$ is the first point. If $\gamma \subset \partial G+\bar{B}\left(3 R_{1}\right)$, the assertion follows from the Cigar theorem 2.29, since $3 R_{1}<R / 4 M_{1}$. Hence we may assume that $\delta(x)>3 R_{1}$ for some $x \in \gamma$. Let $x_{0}$ and $x_{1}$ be the first and the last point of $\gamma$ with $\delta\left(x_{0}\right)=\delta\left(x_{1}\right)=3 R_{1}$. Set $\gamma_{j}=\gamma\left[a_{j}, x_{j}\right]$ for $j=0,1$. We first show that there is $c_{0}=c_{0}(c, \psi) \geq 1$ such that

$$
l\left(\gamma\left[a_{j}, x\right]\right) \leq c_{0} \delta(x)
$$

for all $x \in \gamma_{j}, j=0,1$.

Let $q=q(c, 0, \psi) \in(0,1)$ be the number given by the Diving lemma 2.15. If $\delta(x) \leq q \delta\left(x_{0}\right)=3 q R_{1}$, then (2.36) follows from 2.15 with $c_{0}=4 M_{1} / q$. Assume that $\delta(x) \geq 3 q R_{1}$. Since the Escape lemma 2.13 gives

$$
l\left(\gamma_{j}\right) \leq 12 M_{1} R_{1},
$$

(2.36) again holds with $c_{0}=4 M_{1} / q$.

For $j=0,1$, let $b_{j} \in \gamma$ be the point with $l\left(\gamma\left[a_{j}, b_{j}\right]\right)=t$. Since $t \leq R_{1}$, we have $b_{j} \in \gamma_{j}$. Since $(2.36)$ gives $\delta\left(b_{j}\right) \geq t / c_{0}$ and since

$$
e\left(b_{0}, b_{1}\right) \leq \lambda\left(b_{0}, a_{0}\right)+e\left(a_{0}, a_{1}\right)+\lambda\left(a_{1}, b_{0}\right) \leq 3 t<R,
$$

we get $r_{G}\left(b_{0}, b_{1} ; e\right) \leq 3 c_{0}$ and $k\left(b_{0}, b_{1}\right) \leq \psi\left(3 c_{0}\right)$. Let $c_{2}=c_{1}\left(c, C_{1}\right)$ be the constant of Lemma 2.31 with $C_{1}=\psi\left(3 c_{0}\right)$. Then the $\operatorname{arc} \beta=\gamma\left[b_{0}, b_{1}\right]$ is $c_{2}$-uniform in $G$. Hence $l(\beta) \leq c_{2}\left|b_{0}-b_{1}\right| \leq c_{2} e\left(b_{0}, b_{1}\right) \leq 3 c_{2} t$, and we obtain the turning condition

$$
l(\gamma) \leq\left(2+3 c_{2}\right) t .
$$

To prove the cigar condition, let $x \in \gamma$. If $x \in \gamma_{0} \cup \gamma_{1}$, then (2.36) implies the cigar condition $\zeta_{l}(x, \gamma) \leq c_{0} \delta(x)$. Assume that $x \in \gamma_{2}=\gamma\left[x_{0}, x_{1}\right]$. By (2.37) we have

$$
e\left(x_{0}, x_{1}\right) \leq \lambda\left(x_{0}, a_{0}\right)+e\left(a_{0}, a_{1}\right)+\lambda\left(a_{1}, x_{1}\right) \leq 24 M_{1} R_{1}+R_{1} \leq 25 M_{1} R_{1}=R .
$$

Hence $r_{G}\left(x_{0}, x_{1} ; e\right)<9 M_{1}$, which implies that $k\left(x_{0}, x_{1}\right)<\psi\left(9 M_{1}\right)$. By 2.31, $\gamma_{2}$ is $c_{3}$-uniform in $G$ with $c_{3}=c_{1}\left(c, \psi\left(9 M_{1}\right)\right)$. We show that

$$
c_{3} \delta(x) \geq R_{1}
$$

for all $x \in \gamma_{2}$. If $\left|x-x_{0}\right| \wedge\left|x-x_{1}\right| \geq R_{1}$, this follows from the cigar condition for $\gamma_{2}$. If $\left|x-x_{j}\right| \leq R_{1}$, for $j=0$ or $j=1$, then $c_{3} \delta(x) \geq \delta(x) \geq \delta\left(x_{j}\right)-R_{1}=2 R_{1}>R_{1}$. In view of (2.38) we get

$$
\zeta_{l}(x, \gamma) \leq l(\gamma) / 2<\left(1+2 c_{2}\right) R_{1} \leq\left(1+2 c_{2}\right) c_{3} \delta(x) .
$$

2.39. Theorem. Suppose that $G$ is $R$-boundedly $\mathrm{QH}(\psi, e)$-uniform with a slow $\psi$. Then there are $M_{2}=M_{2}(\psi) \geq 1$ and $c=c(\psi) \geq 2$ such that $G$ is $R_{1}$-boundedly $(c, e)$-uniform with $R_{1}=R / M_{2}$. 
Proof. Since each pair of points in $G$ can be joined by a 2-neargeodesic, this follows from 2.35 with $M_{2}=25 M_{1}(2,0, \psi)$ and $c=c_{1}(2, \psi)$.

2.40. Theorem. For a domain $G$ and for a metric $e$, the following conditions are quantitatively equivalent:

(1) $G$ is R-boundedly (c,e)-uniform.

(2) $G$ is R-boundedly QH $(c, e)$-uniform.

(3) $G$ is $R$-boundedly $\mathrm{QH}(\psi, e)$-uniform with a slow function $\psi$.

Proof. Trivially (2) implies (3) with the same $R$ and with $\psi(t)=c \log (1+t)$. By 2.39, (3) implies (1) with $R \mapsto R / M_{2}(\psi)$ and $c=c(\psi)$. By 2.27, (1) implies (2) with the same $R$ and with $c \mapsto 7 c^{3}$.

2.41. Enlarging the domain. Suppose that $G \subset D$ are proper subdomains of $E$. A neargeodesic in $G$ need not be a neargeodesic in $D$. We next show that this is true if $G$ is a uniform domain, and that this property in fact characterizes uniform domains. Contrary to the theory hitherto, this result seems to have no inner version.

2.42. Theorem. For a domain $G$, the following conditions are quantitatively equivalent:

(1) $G$ is c-uniform.

(2) If $c_{0} \geq 1$ and if $\gamma$ is a $c_{0}$-neargeodesic in $G$, then $\gamma$ is a $c_{1}$-neargeodesic in every domain $D \supset G$ with $c_{1}=c_{1}\left(c_{0}\right)$.

(3) If $\gamma$ is a 2-neargeodesic in $G$ and if $y \in E \backslash G$, then $\gamma$ is a c-neargeodesic in $E \backslash\{y\}$.

Proof. Trivially (2) implies (3) with $c=c_{1}(2)$. It suffices to prove the quantitative implications $(3) \Rightarrow(1) \Rightarrow(2)$.

$(1) \Rightarrow(2)$ : Suppose that $\gamma$ is a $c_{0}$-neargeodesic in $G$ and that $G \subset D \varsubsetneqq E$. Each subarc $\beta$ of $\gamma$ is also a $c_{0}$-neargeodesic in $G$. By the Cigar theorem 2.29 (and by $2.34), \beta$ is $c_{2}$-uniform in $G$ with a constant $c_{2}=c_{2}\left(c, c_{0}\right)$. The turning condition implies that $\gamma$ is $c_{2}$-quasiconvex in the norm metric. By $2.26, \gamma$ is a $7 c_{2}^{3}$-neargeodesic in $D$.

$(3) \Rightarrow(1)$ : Let $a, b \in G$. Choose a 2-neargeodesic $\gamma$ joining $a$ and $b$ in $G$. Let $y \in E \backslash G$. Since $\gamma$ is a $c$-neargeodesic in $D=E \backslash\{y\}$ and since $D$ is a $c^{\prime}$-uniform domain with a universal $c^{\prime}$ by [Vä6,6.5], $\gamma$ is $c_{2}$-uniform in $D$ with $c_{2}=c_{2}(c)$ by 2.29. Since this holds for all $y \in E \backslash G$, the $\operatorname{arc} \gamma$ is $c_{2}$-uniform in $G$.

2.43. Subinvariance. It is well known that uniform domains in $\mathrm{R}^{n}$ are subinvariant under quasiconformal maps. By this we mean that if $f: G \rightarrow G^{\prime}$ is a $K$ quasiconformal map between domains in $\mathrm{R}^{n}$, and if $D \subset G$ and $G^{\prime}$ are $c$-uniform, then $f D$ is $c^{\prime}$-uniform with $c^{\prime}=c^{\prime}(c, K, n)$. This follows from the corresponding result for QED domains [FHM, p. 121] and from [Vä2, 5.6]. An alternative proof is based on the fact that the uniform domains in $\mathrm{R}^{n}$ are precisely the BMO extension domains.

We do not know whether the corresponding result holds for freely quasiconformal maps in Banach spaces. However, we can use 2.42 to prove that uniform domains are subinvariant under QH maps. Recall that a homeomorphism $f: G \rightarrow G^{\prime}$ is $M$-QH if $f$ is $M$-bilipschitz in the QH metric.

2.44. Theorem. Suppose that $f: G \rightarrow G^{\prime}$ is $M-\mathrm{QH}$, that the domain $G^{\prime} \subset E^{\prime}$ is $c$-uniform, and that $D \subset G$ is c-uniform. Then $f D$ is $c^{\prime}$-uniform with $c^{\prime}=c^{\prime}(c, M)$. 
Proof. Let $\gamma$ be a 2-neargeodesic in $f D$, and let $y \in E^{\prime} \backslash f D$. By 2.42, it suffices to show that $\gamma$ is a $c^{\prime}$-neargeodesic in $E^{\prime} \backslash\{y\}$ with $c^{\prime}=c^{\prime}(c, M)$. We let $c_{1}, c_{2}, \ldots$ denote constants depending only on $(c, M)$. The restriction $f_{D}: D \rightarrow f D$ of $f$ is $4 M^{2}$-QH by [Vä5, 4.7]. Hence the arc $\alpha=f^{-1} \gamma$ is a $c_{1}$-neargeodesic in $D$ with $c_{1}=32 M^{4}$. By $2.42, \alpha$ is a $c_{2}$-neargeodesic in $G \backslash f^{-1}\{y\}$. Hence $\gamma$ is a $c_{3}$ neargeodesic in $G^{\prime} \backslash\{y\}$ with $c_{3}=16 M^{4} c_{2}$. Since $G^{\prime} \backslash\{y\}$ is $c_{4}$-uniform by [Vä6, 6.7], $\gamma$ is a $c_{5}$-neargeodesic in $E^{\prime} \backslash\{y\}$ by 2.42 .

2.45. Coarse cigar theorem. Suppose that $G$ is $\mathrm{QH}$ inner $\psi$-uniform rel $A$ with a slow $\psi$ and that $\gamma \subset A$ is a $(c, h)$-solid arc in $G$ with endpoints $a_{0}, a_{1}$. Then

(1) $\operatorname{cig}_{d}\left(\gamma, c_{1}\right) \subset G$,

(2) $d(\gamma) \leq c_{1}\left|a_{0}-a_{1}\right| \vee 2 r\left(e^{h}-1\right)$,

where $c_{1}=c_{1}(c, h, \psi)$ and $r=\delta\left(a_{0}\right) \wedge \delta\left(a_{1}\right)$. This is also true if $G$ is R-boundedly $\mathrm{QH}$ inner $\psi$-uniform and $\gamma \subset G \cap\left(\partial G+\bar{B}\left(R / 4 M_{1}\right)\right)$, where $M_{1}=M_{1}(c, h, \psi)$ is defined by (2.8).

Proof. The first part is again proved almost verbatim as the absolute case in [Vä6, 6.22]. Note, however, that [Vä6, 6.22] has unnecessary parentheses in (2). The second part follows from the first part and from 2.13, which gives $d(\gamma) \leq R$.

2.46. Uniform spaces. Suppose that $X$ is a metric space. If $\gamma \subset X$ is a rectifiable compact arc, we can define $\operatorname{cig}_{l}(\gamma, c)$ as in 2.16. We say that $X$ is a $c$-uniform space if each pair of points in $X$ can be joined by an arc $\gamma$ such that the closure of $\operatorname{cig}_{l}(\gamma, c)$ is complete.

If $e$ is a metric of a domain $G \subset E$ such that $d \mid G \leq e \leq \lambda_{G}$ and if $(G, e)$ is a $c$-uniform space, then $G$ is a $c$-uniform domain in the sense of 2.16. Conversely, if $G$ is a $c$-uniform domain, then $(G, e)$ is a $c^{\prime}$-uniform space for all $c^{\prime}>c$.

\section{Finite-dimensional spaces}

3.1. Summary. In this section we mainly consider the case $\operatorname{dim} E=n<\infty$, with the exception of 3.12. Remember that we always assume that $n \geq 2$. By a result of F. John (see [MiS, 3.3]), there is a linear bijection $f: E \rightarrow R^{n}$ with $|x| \leq|f x| \leq$ $\sqrt{n}|x|$ for all $x \in E$. Hence we shall assume without an essential loss of generality that $E=R^{n}$.

It is well known that uniform domains in $R^{n}$ can be characterized in terms of diameter and distance cigars. With rather obvious modifications, the proofs extend to $(c, e)$-uniform domains, in particular, to inner uniform domains. We also show that in the turning condition, one can replace the inner length metric $\lambda_{G}$ by the inner diameter metric $\varrho_{G}$. In fact, we show that the inner metrics $\varrho_{G}$ and $\lambda_{G}$ are bilipschitz equivalent in a class of domains, called airy domains, which contains all John domains. We also show that the uniform domains are precisely the domains which remain inner uniform under all Möbius maps.

For simplicity, we do not formulate relative and bounded versions of these results.

3.2. Airy sets. We say that a set $A \subset E$ is c-airy, $c \geq 1$, if for each $a \in A$ and $0<t<d(A) / 2$ there is a rectifiable arc $\gamma \subset A$ joining $a$ to a point $b$ such that $l(\gamma) \leq t$ and $B(b, t / c) \subset A$.

John domains, and hence inner uniform domains are airy by Lemma 3.3 below. The planar domain $\{(x, y): y \neq 0$ or $0<x<1\}$ is 1-airy but not a John domain.

3.3. Lemma. A c-John domain is 2c-airy. 
Proof. Let $a \in G$ and let $0<t<d(G) / 2$. Then there is $b \in G$ with $|a-b|>t$. Since $G$ is $c$-John, we can join $a$ and $b$ by an $\operatorname{arc} \gamma$ with $\operatorname{cig}_{l}(\gamma, c) \subset G$. Since $l(\gamma) \geq|a-b|>t$, there is $x \in \gamma$ with $l(\gamma[a, x])=t / 2$, and then $\delta(x) \geq t / 2 c$.

3.4. Theorem. Suppose that $G \subset R^{n}$ is a c-airy domain. Then $\lambda_{G} \leq c^{\prime} \varrho_{G}$ with $c^{\prime}=c^{\prime}(c, n)$.

Proof. Let $a, b \in G$, and set $M=\varrho_{G}(a, b)$. We must find an $\operatorname{arc} \gamma: a \curvearrowright b$ with $l(\gamma) \leq c^{\prime} M$.

Choose an $\operatorname{arc} \alpha: a \curvearrowright b$ with $d(\alpha)<2 M$ and successive points $a=x_{0}, \ldots, x_{N}=$ $b$ of $\alpha$ such that $\left[x_{j-1}, x_{j}\right] \subset G$ for all $j$. Setting $t=M / 2$ we have $0<t<d(G) / 2$. Since $G$ is $c$-airy, we can join each $x_{j}$ to a point $y_{j}$ by an arc $\alpha_{j}$ such that

$$
l\left(\alpha_{j}\right) \leq t, \quad \delta\left(y_{j}\right) \geq t / c .
$$

Set $Y=\left\{y_{j}: 0 \leq j \leq N\right\}$ and $U=B(Y, t / c)$. Let $\mathcal{P}$ be the family of all components of $U$.

Fact $1 . \# \mathcal{P} \leq(6 c)^{n}$.

To prove this, observe that the volume of each member of $\mathcal{P}$ is at least $\Omega_{n}(M / 2 c)^{n}$, where $\Omega_{n}$ is the volume of the unit $n$-ball. Since $\mathcal{P}$ is disjoint, this implies that $m(U) \geq(\# \mathcal{P}) \Omega_{n}(M / 2 c)^{n}$. On the other hand, $U$ is contained in the ball $B(a, R)$ with

$$
R=d(\alpha)+t+t / c \leq 2 M+M / 2+M / 2=3 M .
$$

Hence $m(U) \leq \Omega_{n}(3 M)^{n}$, and Fact 1 follows.

Fact 2. If $V \in \mathcal{P}$ and if $u, v \in V \cap Y$, then there is an $\operatorname{arc} \beta: u \curvearrowright v$ in $V$ such that $l(\beta) \leq c_{1} M$ with a constant $c_{1}=c_{1}(c, n)$.

For $z \in Y \cap V$ we write $B(z)=B(z, t / c)$. Since $V$ is connected, there is a finite sequence $u=z_{0}, \ldots, z_{k}=v$ in $Y \cap V$ such that $B\left(z_{j-1}\right)$ meets $B\left(z_{j}\right)$ for all $1 \leq j \leq k$. Passing to a subsequence we may assume that $B\left(z_{i}\right)$ and $B\left(z_{j}\right)$ are disjoint whenever $|i-j| \geq 2$. Then the balls $B\left(z_{j}\right)$ with even $j$ are disjoint. Since the number of these balls is at least $k / 2$, we get

$$
(k / 2) \Omega_{n}(t / c)^{n} \leq m(V) \leq m(U) \leq \Omega_{n}(3 M)^{n},
$$

and hence $k \leq 2(6 c)^{n}$. Now the union of the segments $\left[z_{j-1}, z_{j}\right]$ is an $\operatorname{arc} \beta: u \curvearrowright v$ with

$$
l(\beta) \leq 2 t k / c=k M / c \leq c_{1} M,
$$

where $c_{1}=2 \cdot 6^{n} c^{n-1}$, and Fact 2 is proved.

We define integers $-1=j_{0}<j_{1}<\cdots<j_{s}=N$ and distinct members $V_{1}, \ldots, V_{s}$ of $\mathcal{P}$ as follows. Let $V_{1}$ be the member containing $y_{0}$, and let $j_{1}$ be the largest number $j$ such that $y_{j} \in V_{1}$. If $j_{1}=N$, then $s=1$ and the process stops. Otherwise we let $V_{2} \in \mathcal{P}$ be the set containing $y_{j_{1}+1}$. We set $j_{2}=\max \left\{j: y_{j} \in V_{2}\right\}$ and continue in this manner until we obtain $j_{s}=N$.

For each $1 \leq i \leq s$ we apply Fact 2 to find an $\operatorname{arc} \beta_{i} \subset V_{i}$ joining the points $y_{j_{i-1}+1}$ and $y_{j_{i}}$. Let $\gamma_{0}$ be the union of these arcs, the line segments $\left[x_{j_{i}}, x_{j_{i}+1}\right], 1 \leq$ $i \leq s-1$, and the $\operatorname{arcs} \alpha_{r}$ for $r=0, j_{1}, j_{1}+1, j_{2}, j_{2}+1, \ldots, j_{s}=N$. Then $\gamma_{0}$ is connected and contains $a$ and $b$. Since $s \leq(6 c)^{n}$ by Fact 1 , the total length of all these arcs is at most

$$
s c_{1} M+s d(\alpha)+2 s t \leq(6 c)^{n}\left(3+c_{1}\right) M=c^{\prime} M .
$$


Hence $\gamma_{0}$ contains an arc $\gamma: a \curvearrowright b$ with $l(\gamma) \leq c^{\prime} M$.

3.5. Theorem. Suppose that $G \subset R^{n}$ is an inner c-uniform domain. Then there is $c_{1}=c_{1}(c, n)$ such that $G$ is $\left(c_{1}, e\right)$-uniform for all metrics e with $\varrho_{G} \leq e \leq \lambda_{G}$.

Proof. As a $c$-John domain, $G$ is $2 c$-airy by 3.3. Hence $\lambda_{G} \leq c^{\prime} \varrho_{G}$, where $c^{\prime}=$ $c^{\prime}(2 c, n)$ is given by 3.4. Consequently, the theorem holds with $c_{1}=c^{\prime}$.

3.6. Example. We show that 3.4 does not hold in the Hilbert space $E=l_{2}$. Let $e_{1}, e_{2}, \ldots$ be the orthonormal basis of $E$, and let $Z$ be the union of all segments $\left[e_{j}, e_{j+1}\right], j \in \mathrm{N}$. Then the neighborhood $G=B(Z, 1 / 10)$ is airy, and $\varrho_{G}(G)<$ $\infty, \lambda_{G}(G)=\infty$.

A more convincing example is given by the union $D$ of this $G$ and all cones $V_{j}=\left\{x: d\left(x, L_{j}\right)<\left|x-e_{j}\right| / 2\right\}, j \in \mathrm{N}$, where $L_{j}=\left\{t e_{j}: t \geq 1\right\}$. Now $D$ is airy and unbounded, $\varrho_{D}\left(e_{1}, e_{j}\right)=\sqrt{2}$, and $\lambda_{D}\left(e_{1}, e_{j}\right) \rightarrow \infty$ as $j \rightarrow \infty$.

3.7. Lemma. A c-John domain $G \subset E$ satisfies the condition $(2 c+1)-\mathrm{LLC}_{2}$ : If $x_{0} \in G$ and $r>0$, then each pair of points in $G \backslash B\left(x_{0},(2 c+1) r\right)$ can be joined by an arc in $G \backslash B\left(x_{0}, r\right)$.

Proof. Let $a, b \in G \backslash B\left(x_{0},(2 c+1) r\right)$ and choose an $\operatorname{arc} \alpha: a \curvearrowright b$ with $\operatorname{cig}_{l}(\alpha, c) \subset G$. If there is $x \in \alpha \cap B\left(x_{0}, r\right)$, then $\zeta_{l}(x, \alpha)>2 c r$, and hence $\delta(x)>2 r$. This implies that $\delta\left(x_{0}\right)>r$, and we obtain the desired arc by replacing a subarc of $\alpha$ by an arc in $S\left(x_{0}, r\right)$.

3.8. Bounded turning. A domain $G$ is of c-bounded turning if each pair $a, b \in G$ can be joined by an $\operatorname{arc} \gamma \subset G$ such that $d(\gamma) \leq c|a-b|$. Then $\varrho_{G} \leq c d \mid G$.

3.9. Theorem. For a domain $G \subset R^{n}$, the following conditions are $n$-quantitatively equivalent:

(1) $G$ is c-uniform.

(2) $G$ is inner c-uniform and of c-bounded turning.

(3) If $g$ is a Möbius map of $R^{n} \cup\{\infty\}$ with $g G \subset R^{n}$, then $g G$ is inner c-uniform.

Proof. By $n$-quantitativeness we mean that the constants $c$ in the conditions depend only on each other and on $n$.

$(1) \Rightarrow(3)$ follows from the fact that a Möbius image of a $c$-uniform domain is $c_{1}$-uniform with $c_{1}=c_{1}(c)$ (see [Vä6, 6.24]).

$(2) \Rightarrow(1)$ : By $3.5, G$ is $\left(c_{1}, \varrho_{G}\right)$-uniform with $c_{1}=c_{1}(c, n)$. Since $\varrho_{G} \leq c d \mid G, G$ is $c c_{1}$-uniform.

$(3) \Rightarrow(2)$ : Trivially, $G$ is inner $c$-uniform. To prove the bounded turning let $a, b \in G$. We may assume that there is $x_{0} \in[a, b] \backslash G$. Set $r=\left|a-x_{0}\right| \vee\left|b-x_{0}\right|$ and let $g$ be the inversion in the sphere $S\left(x_{0}, r\right)$. Since $g G$ is inner $c$-uniform and hence $c$-John, it follows from 3.7 that there is an arc $\alpha: g a \curvearrowright g b$ in $g G \backslash B\left(x_{0}, r / c_{1}\right)$ where $c_{1}=2 c+1$. Then $g \alpha: a \curvearrowright b$ in $G \cap \bar{B}\left(x_{0}, c_{1} r\right)$, and $d(g \alpha) \leq 2 c_{1} r \leq 2 c_{1}|a-b|$. Hence $G$ is of $2 c_{1}$-bounded turning.

3.10. Diameter and distance cigars. Let $\gamma \subset E$ be an arc with endpoints $a$ and $b$. For $c \geq 1$, the length $\operatorname{cigar} \operatorname{cig}_{l}(\gamma, c)$ and the diameter $\operatorname{cigar} \operatorname{cig}_{d}(\gamma, c)$ were defined in 2.16; the former is only defined if $\gamma$ is rectifiable. In addition, we consider the distance cigar

$$
\operatorname{cig}_{\text {dist }}(\gamma, c)=\bigcup\{B(x,(|x-a| \wedge|x-b|) / c): x \in \gamma\} .
$$


We always have

$$
\operatorname{cig}_{\operatorname{dist}}(\gamma, c) \subset \operatorname{cig}_{d}(\gamma, c) \subset \operatorname{cig}_{l}(\gamma, c) .
$$

It is well known that uniform domains in $R^{n}$ can be characterized in terms of diameter cigars [Ma, 4.5] or distance cigars [Vä3, 2.10]. The turning condition $l(\gamma) \leq c|a-b|$ is then replaced by the inequality $d(\gamma) \leq c|a-b|$. In arbitrary Banach spaces, diameter and distance cigars still give the same class of domains, but this is strictly larger than the class of uniform domains. For example, the domain $G \subset l_{2}$ of Example 3.6 is not uniform although it satisfies the corresponding condition with diameter cigars.

We next give the corresponding results for inner uniformity, using the turning condition $d(\gamma) \leq c \varrho_{G}(a, b)$.

3.11. Theorem. For a domain $G \subset R^{n}$, the following conditions are $n$-quantitatively equivalent:

(1) $G$ is inner c-uniform.

(2) Each pair of points $a, b \in G$ can be joined by an arc $\gamma$ such that $\operatorname{cig}_{d}(\gamma, c) \subset G$ and $d(\gamma) \leq c \varrho_{G}(a, b)$.

Proof. Suppose that (1) is true and that $a, b \in G$. By 3.4 there is $c_{1}=c_{1}(c, n)$ such that $\lambda_{G} \leq c_{1} \varrho_{G}$. Let $\gamma: a \curvearrowright b$ be an arc satisfying the $c$-uniformity conditions in $G$. Since $\operatorname{cig}_{d}(\gamma, c) \subset \operatorname{cig}_{l}(\gamma, c)$ and since

$$
d(\gamma) \leq l(\gamma) \leq c \lambda_{G}(a, b) \leq c c_{1} \varrho_{G}(a, b),
$$

(2) holds with the constant $c c_{1}$.

Conversely, assume that (2) is true and that $a, b \in G$. Choose an arc $\gamma: a \curvearrowright b$ satisfying (2). Using a straightening technique of Martio and Sarvas [MaS, 2.7] we can replace $\gamma$ by an inscribed polygonal arc $\alpha$ such that $\operatorname{cig}_{l}\left(\alpha, c_{2}\right) \subset \operatorname{cig}_{d}(\gamma, c)$ and $l(\alpha) \leq c_{2} d(\gamma)$ with some constant $c_{2}=c_{2}(c, n)$. Now $l(\alpha) \leq c_{2} d(\gamma) \leq c c_{2} \varrho_{G}(a, b) \leq$ $c c_{2} \lambda_{G}(a, b)$, and hence (1) holds with the constant $c c_{2}$.

3.12. Theorem. For a domain $G \subset E$, the following conditions are quantitatively equivalent:

(1) Each pair of points $a, b \in G$ can be joined by an arc $\gamma$ such that $\operatorname{cig}_{d}(\gamma, c) \subset G$ and $d(\gamma) \leq c \varrho(a, b)$.

(2) Each pair of points $a, b \in G$ can be joined by an arc $\gamma$ such that $\operatorname{cig}_{\text {dist }}(\gamma, c) \subset$ $G$ and $d(\gamma) \leq c \varrho(a, b)$.

Proof. Observe that contrary to the other results of this section, the theorem holds in all Banach spaces $E$.

Trivially (1) implies (2) with the same $c$. Conversely, assume that (2) is true. Since the argument is well known (see [Vä3, 2.18]), we omit some details. Let $a, b \in$ $G$, and set $2 r=\delta(a) \wedge \delta(b), M=\varrho_{G}(a, b)$. Fix points $a_{1} \in S(a, r), b_{1} \in S(b, r)$. If $M \leq 2 r$, we can choose $\gamma=[a, b]$. If $2 r \leq M \leq 12 r$, we choose an $\operatorname{arc} \beta: a_{1} \curvearrowright b_{1}$ satisfying (2). Then $\gamma=\left[a, a_{1}\right] \cup \beta \cup\left[b, b_{1}\right]$ satisfies in the obvious sense the path version of (1) with the constant $72 c$.

We can thus assume that $M \geq 12 r$. Since $d(G)>\varrho_{G}(a, b)=M$, there is $z \in G$ with $|z-a| \wedge|z-b|>M / 4$. Let $N \geq 2$ be the unique integer with $3 \cdot 2^{N} r \leq M<3 \cdot 2^{N+1} r$. Set $a_{0}=a, A_{1}=\left[a_{0}, a_{1}\right]$, and choose inductively points $a_{j}$ and $\operatorname{arcs} A_{j}: a_{j-1} \curvearrowright a_{j}$ for $1 \leq j \leq N$ as follows: Assuming that $a_{i} \in S\left(a, 2^{i-1} r\right)$ and $A_{i} \subset \bar{B}\left(a, 2^{i-1} r\right)$ have been defined for $i \leq j$, we choose an arc $\alpha_{j}: a_{j} \curvearrowright z$ satisfying (2) and let $a_{j+1}$ be the first point of $\alpha_{j}$ in $S\left(a, 2^{j} r\right)$; then 
$A_{j+1}=\alpha_{j}\left[a_{j}, a_{j+1}\right]$. A similar construction gives points $b_{j} \in S\left(b, 2^{j-1} r\right)$ and arcs $B_{j}: b_{j-1} \curvearrowright b_{j}, 1 \leq j \leq N$. Choosing an $\operatorname{arc} \beta: a_{N} \curvearrowright b_{N}$ satisfying (2) we get in the obvious way a path $\gamma$ joining $a$ and $b$ in $A_{1} \cup \cdots \cup A_{N} \cup \beta \cup B_{N} \cup \cdots \cup B_{1}$. This satisfies the path version of (1) with the constant $96 c^{3}$.

3.13. Remark. Balogh and Volberg [BV1] say that a domain $G \subset R^{2}$ is uniformly John if there is $c$ such that condition (2) of 3.12 holds. By 3.11 and 3.12 we see that for domains $G \subset R^{n}$, this is $n$-quantitatively equivalent to inner $c$-uniformity.

3.14. Remark. Jones [Jo2] says that a domain $G \subset R^{n}$ is an $(\varepsilon, \delta)$ domain with $\varepsilon>0, \delta>0$ if each pair $a, b \in G$ with $|a-b|<\delta$ can be joined by a rectifiable arc $\gamma$ such that $l(\gamma) \leq|a-b| / \varepsilon$ and

$$
\zeta_{J}(x)=\frac{|x-a||x-b|}{|a-b|} \leq d(x, \partial G) / \varepsilon
$$

for all $x \in \gamma$. We show that this property is $n$-quantitatively equivalent to $R$ bounded $c$-uniformity. Setting $\zeta(x)=|x-a| \wedge|x-b|$ we have

$$
1 / \zeta_{J}(x) \leq 1 /|x-b|+1 /|x-a| \leq 2 / \zeta(x)
$$

In the other direction, assuming the turning condition $l(\gamma) \leq c|a-b|$ we get

$$
|x-a| \vee|x-b| \leq l(\gamma) \leq c|a-b|
$$

and hence

$$
\zeta_{J}(x) \leq c \zeta(x) \leq c \zeta_{l}(x) .
$$

From this it follows that an $R$-boundedly $c$-uniform domain is an $(\varepsilon, \delta)$ domain with $\delta=R, \varepsilon=c^{-2}$. Conversely, an $(\varepsilon, \delta)$ domain is $R$-boundedly $c$-uniform with $R=\delta / 2, c=c(\varepsilon, n)$. To see this, let $a, b \in G$ with $|a-b| \leq R$, and let $\gamma: a \curvearrowright b$ be an arc satisfying the $(\varepsilon, \delta)$ condition. Then $\operatorname{cig}_{\text {dist }}(\gamma, 2 / \varepsilon) \subset G$ by (3.15). A variation of the proof of 3.9 gives an $\operatorname{arc} \beta: a \curvearrowright b$ such that $\operatorname{cig}_{d}\left(\beta, c_{1}\right) \subset G$ and $d(\beta) \leq c_{1}|a-b|$ with some $c_{1}=c_{1}(\varepsilon)$; the proof only makes use of pairs with distance less than $2 R=\delta$. Finally, the straightening technique of [MaS, 2.7] again gives the desired $c$-uniform arc $\alpha: a \curvearrowright b$ with $c=c(\varepsilon, n)$.

\section{Boundary EXtension}

4.1. Summary. We study the question: When does a coarsely quasihyperbolic map $f: G \rightarrow G^{\prime}$ have a limit at a given boundary point? This question has been rather extensively studied for quasiconformal maps in $\mathrm{R}^{n}$. In the free quasiworld, it is known that $\mathrm{CQH}$ maps between uniform domains have homeomorphic extensions to the closures. For nonuniform domains, there are hardly any earlier results.

4.2. Definitions. We first recall the basic terminology for maps in the free quasiworld. Let $E$ and $E^{\prime}$ be Banach spaces, and let $G \subset E$ and $G^{\prime} \subset E^{\prime}$ be domains with QH metrics $k=k_{G}$ and $k^{\prime}=k_{G^{\prime}}$. Let $f: G \rightarrow G^{\prime}$ be a homeomorphism. If $M \geq 1, C \geq 0$, and

$$
(k(x, y)-C) / M \leq k^{\prime}(f x, f y) \leq M k(x, y)+C,
$$

then $f$ is said to be $C$-coarsely $M$-quasihyperbolic or briefly $(M, C)$-CQH. The map is $M$-quasihyperbolic if $M$-QH and if this holds with $C=0$. In other words, $f$ is $M$-bilipschitz in the QH metric. If $\varphi:[0, \infty) \rightarrow[0, \infty)$ is a homeomorphism and if

$$
\varphi^{-1}(k(x, y)) \leq k^{\prime}(f x, f y) \leq \varphi(k(x, y))
$$


for all $x, y \in G$, then $f$ is $\varphi$-solid. If $f$ defines a $\varphi$-solid map $f_{D}: D \rightarrow f D$ for each proper subdomain $D \varsubsetneqq G$, then $f$ is freely $\varphi$-quasiconformal. This notion makes sense also in the case $G=E, G^{\prime}=E^{\prime}$. Between these classes, we have the following quantitative implications:

$$
M \text {-QH } \Rightarrow \text { freely } \varphi \text {-quasiconformal } \Rightarrow \varphi \text {-solid } \Rightarrow(M, C) \text {-CQH; }
$$

see [Vä6, 4.14]. Alternatively, the freely quasiconformal maps can be characterized by the property that for some $(M, C)$, each $f_{D}: D \rightarrow f D$ is $(M, C)-\mathrm{CQH}$ [Vä7, 2.21]. In the case $E=E^{\prime}=\mathrm{R}^{n}$, the freely quasiconformal maps are just the quasiconformal maps in the ordinary sense.

We shall consider the boundary behavior of CQH maps. Hence all results hold for the other three classes as well, and for quasiconformal maps of domains in $\mathrm{R}^{n}$.

The closure $\bar{A}$ and the boundary $\partial A$ of a set $A \subset E$ is always taken in the extended space $\dot{E}=E \cup\{\infty\}$, where the neighborhoods of $\infty$ are the complements of closed bounded sets. In particular, $\infty \in \partial A$ if and only if $A$ is unbounded.

Let $c \geq 1$ and $h \geq 0$. A domain $G$ is said to be locally $(c, h)$-solidly connected, or $(c, h)$-LSC, at a point $b \in \partial G$ if each neighborhood $U$ of $b$ contains a neighborhood $V$ of $b$ such that every pair of points in $V \cap G$ can be joined by an $\operatorname{arc} \gamma \subset U \cap G$ such that $\gamma$ is $(c, h)$-solid in $G$.

For example, a Jordan domain $G \subset \mathrm{R}^{2}$ is $(4,0)$-LSC at each boundary point. This is seen by mapping $G$ conformally onto the unit disk and using the fact that hyperbolic geodesics are 4-neargeodesics. More examples of the LSC property are given by the following result:

4.3. Theorem. An R-boundedly c-uniform domain is $\left(c^{\prime}, 0\right)$-LSC at each finite boundary point for all $c^{\prime}>1$. An unbounded c-uniform domain is $\left(c_{0}, 0\right)$-LSC at $\infty$ with a universal constant $c_{0}<145$.

Proof. Suppose that $G$ is $R$-boundedly $c$-uniform. By $2.40, G$ is $R$-boundedly QH $\psi$-uniform with the slow function $\psi=7 c^{3} \log (1+t)$. Let $M_{1}=M_{1}(c, 0, \psi)$ be the number given by (2.11), and let $0<r<R / 50 M_{1}$. Suppose that $1<c^{\prime} \leq 2$, that $b \in \partial G, b \neq \infty$, and that $x, y \in G \cap B(b, r)$. Choose a $c^{\prime}$-neargeodesic $\gamma: x \curvearrowright y$. By 2.35, $\gamma$ is $c_{1}$-uniform in $G$ with $c_{1}=c_{1}(c)$. The turning condition implies that $\gamma \subset B\left(b,\left(c_{1}+1\right) r\right)$. Hence $G$ is $\left(c^{\prime}, 0\right)$-LSC at $b$.

Next assume that $G$ is unbounded and $c$-uniform. We may assume that $0 \in \partial G$. Let $u: E \backslash\{0\} \rightarrow E \backslash\{0\}$ be the inversion $u x=x /|x|^{2}$. Since $u$ is $\eta$-quasimöbius with $\eta(t)=81 t$ by [Vä2, 1.6], the domain $u G$ is uniform by [Vä6, 6.26]. By the first part of the theorem, $u G$ is $\left(c^{\prime}, 0\right)$-LSC at the origin for each $c^{\prime}>1$. By [Vä8, 2.9], $u$ defines a 12-QH map $f: G \rightarrow u G$. Hence $f^{-1}$ maps each $c^{\prime}$-neargeodesic of $u G$ onto a $c_{0}$-neargeodesic of $G$ with $c_{0}=144 c^{\prime}$, and the theorem follows.

4.4. Remarks. 1. If $G$ is $(c, h)$-LSC at $b \in \partial G$, then $G$ is locally connected at $b$, that is, $b$ has arbitrarily small neighborhoods $U$ such that $U \cap G$ is connected. In 4.6 we show that the converse is not true.

2. A parallel strip in $\mathrm{R}^{2}$ is $R$-boundedly $c_{R}$-uniform for all $R>0$, but it is not even locally connected at $\infty$. However, domains $G_{0}=E_{1} \times B_{2}$ are LSC at $\infty$, where $B_{2}$ is a ball in a space $E_{2}$ and $\operatorname{dim} E_{1} \geq 2$. The proof will be given in 5.11. For example, a domain between two parallel planes in $R^{3}$ is LSC at $\infty$.

3. We get more examples of the LSC property by auxiliary maps and by the following result: 
4.5. Theorem. Suppose that $f: G \rightarrow G^{\prime}$ in $(M, C)-\mathrm{CQH}$ and that $b \in \partial G$ and $b^{\prime} \in \partial G^{\prime}$ are points such that $f$ extends to a homeomorphism $G \cup\{b\} \rightarrow G^{\prime} \cup\left\{b^{\prime}\right\}$. If $G$ is $(c, h)$-LSC at $b$, then $G^{\prime}$ is $\left(c^{\prime}, h^{\prime}\right)$-LSC at $b^{\prime}$ with $\left(c^{\prime}, h^{\prime}\right)$ depending only on $(c, h, M, C)$.

Proof. Since $f$ maps $(c, h)$-solid arcs onto $\left(c^{\prime}, h^{\prime}\right)$-solid arcs by [Vä6, 4.15], the result follows from the definition of the LSC property.

4.6. Example. We construct a domain $G \subset \mathrm{R}^{3}$, which is locally connected at $0 \in \partial G$ but not LSC at 0 . Define $g:(0,1] \rightarrow \mathrm{R}$ by $g(t)=e^{-1 / t}$, and set $A=$ $\bar{B}^{2} \backslash\{(x, y): 0<x \leq 1,|y|<g(x)\}$. The domain $G=\mathrm{R}^{3} \backslash A$ is locally connected at the origin.

Let $c \geq 1$ and $h \geq 0$. Then there is $r=r(c, h)>0$ such that no arc in $B(r) \cap G$ is $(c, h)$-solid and joins a point in $\left[0, e_{3}\right]$ to a point in $\left[0,-e_{3}\right]$. We omit the elementary but lengthy proof. Thus $G$ is not $(c, h)$-LSC at the origin.

4.7. Remark. Fattening slightly the set $A$ in 4.6 we obtain an example where $A$ is a topological 3-cell. With a Möbius map we get a Jordan domain in $\mathrm{R}^{3}$ that is not LSC at one boundary point. Recall from 4.2 that Jordan domains in $\mathrm{R}^{2}$ are LSC at all boundary points.

We next give the main result of this section. We let $\lambda^{\prime}=\lambda_{G^{\prime}}$ denote the inner metric of $G^{\prime}$.

4.8. Theorem. Suppose that $f: G \rightarrow G^{\prime}$ is $\mathrm{CQH}$ and that $G$ is LSC at $b \in \partial G$. Suppose also that $d \mid G^{\prime} \leq e \leq \lambda^{\prime}$ and that

(1) for each bounded set $A \subset G^{\prime}$ there is $c_{A} \geq 1$ such that $G^{\prime}$ is $\mathrm{QH}\left(c_{A}, e\right)$ uniform rel $A$,

(2) $e(A)<\infty$ for each bounded $A \subset G^{\prime}$.

Then $f$ has a limit at $b$.

Condition (2) holds automatically if $e=d \mid G^{\prime}$ or if $G^{\prime}$ is bounded or if $\operatorname{dim} E^{\prime}<$ $\infty$

Proof. Suppose that $f$ is $(M, C)-\mathrm{CQH}$ and that $G$ is $(c, h)$-LSC at $b$. Assume that $f$ does not have a limit at $b$.

Suppose first that $b \neq \infty$. For $r>0$ we write $U(r)=B(b, r)$. Fix a point $x_{0} \in G$. Since $\infty$ is not a limit of $f$ at $b$, there is $R>0$ such that $f U(r)$ meets $B\left(f x_{0}, R\right)$ for all $r>0$. Furthermore, there is $t>0$ such that $d(f U(r))>t$ for all $r>0$, since otherwise $\lim _{x \rightarrow b} f(x)$ exists by the completeness of $E^{\prime}$.

Let $r>0$. Since $G$ is $(c, h)$-LSC at $b$, there is $s \in(0, r)$ such that points of $U(s)$ can be joined by a $(c, h)$-solid $\operatorname{arc}$ of $G$ in $U(r)$. Pick $x \in U(s)$ with $f x \in B\left(f x_{0}, R\right)$ and then $y \in U(s)$ with $|f x-f y| \geq t / 2$. Let $\alpha \subset U(r)$ be a $(c, h)$-solid arc in $G$ with endpoints $x$ and $y$. By [Vä6, 4.15], the arc $f \alpha$ is $\left(c^{\prime}, h^{\prime}\right)$-solid in $G^{\prime}$ with $\left(c^{\prime}, h^{\prime}\right)$ depending on $(c, h, M, C)$.

Orient $\alpha$ so that $x$ is its first point. Let $z$ be the first point of $\alpha$ with $|f x-f z|=$ $t / 2$. Let $z_{0} \in \alpha[x, z]$ be a point where $\delta^{\prime}\left(f z_{0}\right)=d\left(f z_{0}, \partial G^{\prime}\right)$ is maximal. Write $r^{\prime}=\delta^{\prime}\left(f z_{0}\right), \gamma=f \alpha[x, z]$, and $A=G^{\prime} \cap B\left(f x_{0}, R+t / 2\right)$. Since $G^{\prime}$ is $\left(c_{A}, e\right)$-uniform rel $A$ and since $\gamma \subset G^{\prime} \cap \bar{B}(f x, t / 2) \subset A$, we can apply 2.27 and the Escape lemma 2.13 with $G \mapsto G^{\prime}, r \mapsto r^{\prime}$ to obtain an estimate $d(\gamma) \leq M_{2} r^{\prime}$ with a constant $M_{2}$ depending on $\left(c, h, M, C, c_{A}\right)$ but not on $r$. Hence $r^{\prime} \geq d(\gamma) / M_{2} \geq t / 2 M_{2}$, which 
yields

$$
r_{G^{\prime}}\left(f x_{0}, f z_{0} ; e\right) \leq \frac{e(A)}{\min \left(t / 2 M_{2}, \delta^{\prime}\left(f\left(x_{0}\right)\right)\right)}=c_{1}<\infty,
$$

by (2), where $c_{1}$ does not depend on $r$. Since $G^{\prime}$ is $\left(c_{A}, e\right)$-uniform rel $A$, and since $f x_{0}, f z_{0} \in A$, this and 2.27 imply that $k^{\prime}\left(f x_{0}, f z_{0}\right) \leq c_{A} \log \left(1+c_{1}\right)=c_{2}$. Since $f$ is $(M, C)-\mathrm{CQH}$, we obtain

$$
k\left(x_{0}, z_{0}\right) \leq M c_{2}+C .
$$

On the other hand,

$$
k\left(x_{0}, z_{0}\right) \geq \log \frac{\delta\left(x_{0}\right)}{\delta\left(z_{0}\right)} \geq \log \frac{\delta\left(x_{0}\right)}{r} .
$$

As $r \rightarrow 0$, these inequalities give a contradiction.

If $b=\infty$, we can use the same argument replacing $U(r)$ by $G \backslash \bar{B}(1 / r)$. For $r<1 /\left|x_{0}\right|$ we then have

$$
k\left(x_{0}, z_{0}\right) \geq \log \frac{\left|x_{0}-z_{0}\right|}{\delta\left(x_{0}\right)} \geq \log \frac{1 / r-\left|x_{0}\right|}{\delta\left(x_{0}\right)} \rightarrow \infty
$$

as $r \rightarrow 0$.

If $e=d \mid G^{\prime}$, then (2) is trivially true. Suppose that $G^{\prime}$ is bounded and that $a, b \in G^{\prime}$. Since $G^{\prime}$ is $\left(c_{0}, e\right)$-uniform with $c_{0}=c_{G^{\prime}}$ by $(1)$, we can join $a$ and $b$ by an $\operatorname{arc} \beta$ such that $\operatorname{cig}_{l}\left(\beta, c_{0}\right) \subset G^{\prime}$. Let $x \in \beta$ be the point with $l(\beta[a, x])=l(\beta) / 2$. Then $B\left(x, l(\beta) / 2 c_{0}\right) \subset G^{\prime}$, which implies that

$$
e(a, b) \leq \lambda^{\prime}(a, b) \leq l(\beta) \leq c_{0} d\left(G^{\prime}\right) .
$$

Hence $e\left(G^{\prime}\right) \leq c_{0} d\left(G^{\prime}\right)<\infty$. If $\operatorname{dim} E^{\prime}<\infty$, then (2) follows from (1) and from Lemma 4.9 below.

4.9. Lemma. Suppose that $\operatorname{dim} E<\infty$, that $G \subset E$ is a domain, that $c \geq 1$, and that $A \subset G$ is a bounded set such that each pair of points in $A$ can be joined by an arc $\gamma$ with $\operatorname{cig}_{l}(\gamma, c) \subset G$. Then $A$ is bounded in the metric $\lambda_{G}$.

Proof. Assume that $\lambda(A)=\infty$. Choose a ball $B(R)$ containing $A$ and a sequence of points $x_{0}, x_{1}, \ldots$ in $A$ such that $\lambda\left(x_{i}, x_{j}\right) \geq 2 R$ for $i \neq j$. For $j \geq 1$ join $x_{j}$ and $x_{0}$ by an $\operatorname{arc} \gamma_{j}$ with $\operatorname{cig}_{l}\left(\gamma_{j}, c\right) \subset G$. Then $l\left(\gamma_{j}\right) \geq 2 R$ for all $j$. Let $y_{j} \in \gamma_{j}$ be the point with $l\left(\gamma\left[x_{j}, y_{j}\right]\right)=R / 4$. Then the balls $B_{j}=B\left(y_{j}, R / 4 c\right)$ lie in $G$. If $B_{i}$ meets $B_{j}$, we can join $x_{i}$ and $x_{j}$ by an $\operatorname{arc} \alpha \subset \gamma\left[x_{i}, y_{i}\right] \cup\left[y_{i}, y_{j}\right] \cup \gamma\left[y_{j}, x_{j}\right] \subset G$, and we obtain the contradiction

$$
\lambda\left(x_{i}, x_{j}\right) \leq l(\alpha) \leq R / 2+R / 2 c \leq R<2 R \leq \lambda\left(x_{i}, x_{j}\right) .
$$

Hence the balls $B_{j}$ are disjoint. Since they lie in $B(2 R)$ and since $\operatorname{dim} E<\infty$, this leads to a contradiction, and the lemma is proved.

4.10. Theorem. Suppose that $G$ and $G^{\prime}$ are domains in $R^{n}$ with $G^{\prime}$ inner uniform, and that $f: G \rightarrow G^{\prime}$ is CQH, for example, quasiconformal. If $G$ is LSC at $b \in \partial G$, then $f$ has a limit at $b$. If $G$ is uniform, then $f$ has a continuous extension to $\bar{G}$.

Proof. This follows from 4.8 and 4.3.

4.11. Theorem. Suppose that $f: G \rightarrow G^{\prime}$ is $\mathrm{CQH}$, that $G$ is $\mathrm{LSC}$ at $b \in \partial G$, and that $G^{\prime}$ is convex. Then $f$ has a limit at $b$. 
Proof. Let $A \subset G^{\prime}$ be bounded. Choose a ball $B$ containing $A$. The domain $G^{\prime} \cap B$ is uniform by 2.19 , and hence $\mathrm{QH} \psi$-uniform with a slow $\psi$. The theorem follows from 4.8 with $e=d \mid G^{\prime}$.

4.12. Example. We show that Theorem 4.8 does not hold without condition (2). Let $E$ be a separable Hilbert space with orthonormal base $e_{1}, e_{2}, \ldots$ We consider $e_{1}$ as vertical, and let $G$ denote the lower half space $\left\{x: x \cdot e_{1}<0\right\}$. Set $T=\{x$ : $\left.x \cdot e_{1}=0\right\}$. For $k \geq 2$, let $B_{k}$ be the disk $T \cap B\left(4 k e_{k}, k\right)$. Define a 4-bilipschitz function $u: T \rightarrow \mathrm{R}$ by $u(x)=4\left(k-\left|x-4 k e_{k}\right|\right)$ for $x \in B_{k}, k \geq 2$, and by $u(x)=0$ elsewhere. Let $P: E \rightarrow T$ be the orthogonal projection. Define a homeomorphism $f_{1}: G \rightarrow G_{1}$ by $f_{1}(x)=x+u(P x) e_{1}$. The image domain $G_{1}$ is the union of $G$, the disks $B_{k}$, and the conical domains $V_{k}$ with base $B_{k}$ and vertex $v_{k}=4 k\left(e_{k}+e_{1}\right)$. Since $f^{-1}(x)=x-u(P x) e_{1}$, we see that $f_{1}$ is 5 -bilipschitz.

For each $k \geq 2$, we can easily find a homeomorphism $g_{k}: \bar{V}_{k} \rightarrow \bar{V}_{k}^{\prime}$ such that $g_{k} \mid B_{k}=\mathrm{id}, g_{k} v_{k}=0$, the domains $V_{k}^{\prime}$ are disjoint subdomains of the upper half space, and the maps $g_{k}$ are $M$-bilipschitz for some $M$ independent of $k$. The maps $g_{k} \mid V_{k}$ extend by identity to a homeomorphism $g: G_{1} \rightarrow G^{\prime}$, where $G^{\prime}$ is the union of $G$ and all $B_{k}$ and $V_{k}^{\prime}$. Since $g$ is locally $M$-bilipschitz, we obtain a locally $5 M$-bilipschitz homeomorphism $f=g \circ f_{1}: G \rightarrow G^{\prime}$. By [Vä5, 4.8], $f$ is $25 M^{2}$-quasihyperbolic. The half space $G$ is uniform. By 2.21 , the domain $G^{\prime}$ is inner uniform. Hence the conditions of 4.8 are satisfied with $e=\lambda^{\prime}$, except for (2). Since $f$ has no limit at $\infty$, we see that condition (2) cannot be omitted.

4.13. Local uniformity. We say that a domain $G$ is locally c-uniform at a point $b \in \partial G$ if $b$ has a neighborhood $U$ such that $G$ is $c$-uniform $\operatorname{rel} U \cap G$, that is, each pair $x, y \in U \cap G$ can be joined by an arc $\gamma$ which is $c$-uniform in $G$.

A $c$-uniform domain is trivially locally $c$-uniform at each boundary point. Conversely, if $G$ is bounded and locally $c$-uniform at each boundary point, then $G$ is a uniform domain. This was proved by P. Alestalo [Al], but the result is not needed in this paper. The domain $G_{0}=E_{1} \times B_{2}$ is locally $c$-uniform with a universal $c$ at each finite boundary point but not at $\infty$. This difference will be used in Section 5 to prove that if $\operatorname{dim} E_{1} \geq 2$, then every CQH map $G_{0} \rightarrow G_{0}$ must fix the point at infinity. The proof is based on 4.15 below.

4.14. Theorem. Suppose that $G$ is locally $c_{0}$-uniform at a finite boundary point $b$. Then there is $r_{0}>0$ such that if $0<r \leq r_{0}$ and if $\gamma$ is a $(c, h)$-solid arc in $G$ with endpoints in $B(b, r)$, then $\gamma \subset B\left(b, M_{2} r\right)$, where $M_{2}$ depends on $\left(c, h, c_{0}\right)$. Moreover, $G$ is $(c, 0)$-LSC at $b$ for each $c>1$.

Proof. Choose a neighborhood $U$ of $b$ such that $G$ is $c_{0}$-uniform rel $U \cap G$. Then $G$ is QH $7 c_{0}^{3}$-uniform rel $U \cap G$ by 2.27 . Let $r_{1}>0$ be such that $\bar{B}\left(b, r_{1}\right) \subset U$, and let $M_{1}=M_{1}(c, h, \psi)$ be the constant given by $(2.11)$ for $\psi(t)=7 c_{0}^{3} \log (1+t)$. We show that the theorem holds with $r_{0}=r_{1} /\left(1+4 M_{1}\right)$ and with a suitable $M_{2}$.

Let $0<r \leq r_{0}$, and let $\gamma$ be a $(c, h)$-solid $\operatorname{arc}$ in $G$ with endpoints $a_{0}, a_{1} \in B(b, r)$. We let $c_{1}, c_{2}, \ldots$ denote constants depending only on $\left(c, h, c_{0}\right)$.

We may assume that $\gamma \not \subset B\left(b,\left(1+4 M_{1}\right) r\right)$, since otherwise the theorem holds with $M_{2}=1+4 M_{1}$. Let $x_{0}$ be the first point of $\gamma$ after $a_{0}$ such that $\left|x_{0}-b\right|=$ $\left(1+4 M_{1}\right) r$. If $\gamma\left[a_{0}, x_{0}\right] \subset \partial G+\bar{B}(r)$, it follows from the Escape lemma 2.13 that $d\left(\gamma\left[a_{0}, x_{0}\right]\right) \leq 4 M_{1} r$. This gives the contradiction

$$
\left|b-x_{0}\right| \leq\left|b-a_{0}\right|+\left|a_{0}-x_{0}\right|<r+4 M_{1} r=\left|b-x_{0}\right| .
$$


Consequently, $\gamma\left[a_{0}, x_{0}\right] \not \subset \partial G+\bar{B}(r)$. Considering similarly the other endpoint $a_{1}$, we find points $b_{0}, b_{1} \in \gamma$ such that $\delta\left(b_{j}\right)=r$ and such that $\gamma\left[a_{j}, b_{j}\right] \subset$ $\bar{B}\left(b,\left(1+4 M_{1}\right) r\right), j=0,1$. Then

$$
r_{G}\left(b_{0}, b_{1}\right)=\frac{\left|b_{0}-b_{1}\right|}{r} \leq 2\left(1+4 M_{1}\right)=c_{1} .
$$

Since $b_{0}, b_{1} \in U$, this implies that $k\left(b_{0}, b_{1}\right) \leq \psi\left(c_{1}\right)$. Setting $\beta=\gamma\left[b_{0}, b_{1}\right]$ we thus have $l_{k}(\beta, h) \leq c \psi\left(c_{1}\right)=c_{2}$.

Let $y \in \beta$. If $k\left(y, b_{0}\right) \leq h$, then [Vä5, 2.2(1)] gives $\left|y-b_{0}\right| \leq \delta\left(b_{0}\right) e^{k\left(y, b_{0}\right)} \leq r e^{h}$. If $k\left(y, b_{0}\right) \geq h$, then

$$
l_{k}(\beta, h) \geq k\left(y, b_{0}\right) \geq \log \frac{\left|y-b_{0}\right|}{r} .
$$

Hence $\left|y-b_{0}\right| \leq r e^{c_{2}}$. Setting $c_{3}=e^{h \vee c_{2}}$ we thus have $\left|y-b_{0}\right| \leq c_{3} r$ in each case. Consequently,

$$
|y-b| \leq\left|y-b_{0}\right|+\left|b_{0}-b\right|<\left(c_{3}+1+4 M_{1}\right) r,
$$

and the first part of the theorem is proved with $M_{2}=c_{3}+1+4 M_{1}$. The second part is a corollary of the first part.

4.15. Theorem. Suppose that $G$ is locally c-uniform at a finite boundary point $b$. Then there is no open solid arc $\gamma \subset G$ such that $\gamma \cup\{b\}$ is a Jordan curve. If $G$ is uniform, this also holds in the case $b=\infty$.

Proof. The first part follows from 4.14. If $G$ is uniform, we can make use of an auxiliary inversion to reduce the case to the first part.

\section{Maps of domains $G_{0}=E_{1} \times B_{2}$}

5.1. Summary. Throughout this section we assume that $E_{1}$ and $E_{2}$ are Banach spaces of dimensions $\geq 1$, and we let $B_{j}$ denote the unit ball of $E_{j}, j=1,2$. Other balls in $E_{j}$ are written as $B_{j}(x, r)$. We set $E=E_{1} \times E_{2}$ and $G_{0}=E_{1} \times B_{2}$. For example, $G_{0}$ may be the infinite tube $\mathrm{R} \times B^{2}$ or the domain $\mathrm{R}^{2} \times B^{1}$ between two parallel planes in $\mathrm{R}^{3}$. In $\mathrm{R}^{n}$, the domain $G_{0}$ is $\mathrm{R}^{p} \times B^{n-p}$ with $1 \leq p \leq n-1$. The author knows of no previous article dealing with the intermediate case $2 \leq p \leq n-2$.

We study CQH maps between these domains. If $G_{0}^{\prime}=E_{1}^{\prime} \times B_{2}^{\prime}$ is another such domain, and if there is a CQH map $f: G_{0} \rightarrow G_{0}^{\prime}$, we show that $\operatorname{dim} E_{1}=\operatorname{dim} E_{1}^{\prime}$ and $\operatorname{dim} E_{2}=\operatorname{dim} E_{2}^{\prime}$. Hence we mainly consider self maps $f: G_{0} \rightarrow G_{0}$. If $\operatorname{dim} E_{1} \geq 2$, then $f$ extends to a homeomorphism $\bar{f}: \bar{G}_{0} \rightarrow \bar{G}_{0}$ with $\bar{f}(\infty)=\infty$. We also show that $\bar{f}$ is quasisymmetric rel $\partial G_{0}$ and estimate the horizontal and vertical distortions of $f$.

5.2. Norm of $E$. We say that a norm $|x|$ of $E=E_{1} \times E_{2}$ is admissible if

$$
\left|x_{1}\right| \vee\left|x_{2}\right| \leq|x| \leq\left|x_{1}\right|+\left|x_{2}\right|
$$

for all $x_{1}, x_{2} \in E$. Throughout this section we assume that the norm of $E$ is admissible. If $E_{1}=\mathrm{R}^{p}$ and $E_{2}=\mathrm{R}^{q}$, we use the euclidean norm $|x|=\left(x_{1}^{2}+x_{2}^{2}\right)^{1 / 2}$, and we identify $E=\mathrm{R}^{p+q}$. Since $\left|x_{1}\right|+\left|x_{2}\right| \leq 2\left(\left|x_{1}\right| \vee\left|x_{2}\right|\right)$, all admissible norms are bilipschitz equivalent.

Let $P_{1}: E \rightarrow E_{1}$ and $P_{2}: E \rightarrow E_{2}$ be the projections. From (5.3) it follows that the operator norms $\left|P_{1}\right|$ and $\left|P_{2}\right|$ are equal to 1 . Moreover, the natural embeddings $E_{1} \rightarrow E$ and $E_{2} \rightarrow E$ are isometries. Hence we can identify $E_{1}$ and $E_{2}$ with the 
subspaces $E_{1} \times\{0\}$ and $\{0\} \times E_{2}$ of $E$. Then we can write $x=P_{1} x+P_{2} x$ for each $x \in E$.

5.4. Quasihyperbolic metric of $G_{0}$. Since $\partial G_{0}=\left(E_{1} \times \partial B_{2}\right) \cup\{\infty\}$, we have $\delta(x)=d\left(x, \partial G_{0}\right)=1-\left|x_{2}\right| \leq 1$ for each $x=\left(x_{1}, x_{2}\right) \in G_{0}$. Let $k=k_{G_{0}}$ be the QH metric of $G_{0}$. We list some obvious properties of $k$. Let $a \in E_{1}$ and $u \in \partial B_{2}$.

(1) If $0 \leq s \leq t<1$, then

$$
k(a+s u, a+t u)=\log \frac{1-s}{1-t} .
$$

In particular, $k(a, a+t u)=\log (1 /(1-t))$.

(2) The segment $[a, a+u)$ is a QH geodesic.

(3) If $a, b \in E_{1}$, then $k(a, b)=|a-b|$.

(4) If $x, y \in G_{0}$ and if $\delta(x), \delta(y) \geq r$, then $r k(x, y) \leq|x-y| \leq k(x, y)$.

(5) Every line $L \subset E_{1}$ is a $\mathrm{QH}$ geodesic of $G_{0}$. If $x \in G_{0}$, the line $L+x$ is a $c$-neargeodesic with $c=1 / \delta(x)$.

5.5. Lemma. Suppose that $\alpha \subset E_{1}$ is a c-quasiconvex arc with endpoints a, $a^{\prime}$ and that $u \in \partial B_{2}$. Then the arc $\gamma=[a, a+u) \cup \alpha$ is a 2c-neargeodesic of $G_{0}$.

Proof. Let $0<t<1$ and set $\gamma_{0}=\alpha \cup[a, a+t u]$. In view of (2) and (3) of 5.4, it suffices to show that

$$
l_{k}\left(\gamma_{0}\right) \leq 2 c k\left(a^{\prime}, a+t u\right) .
$$

Let $\beta: a^{\prime} \curvearrowright a+t u$ be a rectifiable arc in $G_{0}$. Since $\delta(x) \leq 1$ in $G_{0}$, we have $l_{k}(\beta) \geq l(\beta) \geq\left|a-a^{\prime}\right|$. Moreover,

$$
l_{k}(\beta)=\int_{\beta} \frac{|d x|}{1-\left|P_{2} x\right|} \geq \int_{0}^{t} \frac{d r}{1-r}=\log \frac{1}{1-t}
$$

a rigorous proof of the inequality can be carried out by 5.3 and 5.7 of [Vä1]. Hence

$$
2 l_{k}(\beta) \geq\left|a-a^{\prime}\right|+\log \frac{1}{1-t} \geq l(\alpha) / c+\log \frac{1}{1-t} \geq l_{k}\left(\gamma_{0}\right) / c,
$$

and (5.6) follows.

5.7. Lemma. Suppose that $\alpha \subset E_{1}$ is a c-quasiconvex arc with endpoints a, $a^{\prime}$ and that $u, u^{\prime} \in \partial B_{2}$. If $\left|a-a^{\prime}\right| \geq 2$, then $\gamma=[a, a+u) \cup \alpha \cup\left[a^{\prime}, a^{\prime}+u^{\prime}\right)$ is a $2 c$-neargeodesic of $G_{0}$.

Proof. Let $t, t^{\prime} \in[0,1)$ and set $b=a+t u, b^{\prime}=a^{\prime}+t^{\prime} u^{\prime}$. Write $\gamma_{0}=\gamma\left[b, b^{\prime}\right]$ and suppose that $\beta: b \curvearrowright b^{\prime}$ is a rectifiable arc in $G_{0}$. In view of 5.5 , it suffices to show that $l_{k}\left(\gamma_{0}\right) \leq 2 c l_{k}(\beta)$.

Let $x_{0} \in \beta$ be a point where $\delta\left(x_{0}\right)$ is maximal, and set $r=\delta\left(x_{0}\right)$. Then $(1-t) \vee$ $\left(1-t^{\prime}\right) \leq r \leq 1$. For the $\operatorname{arcs} \sigma=\beta\left[b, x_{0}\right]$ and $\sigma^{\prime}=\beta\left[x_{0}, b^{\prime}\right]$ we have as in 5.5

$$
l_{k}(\sigma) \geq \log \frac{r}{1-t}, \quad l_{k}\left(\sigma^{\prime}\right) \geq \log \frac{r}{1-t^{\prime}} .
$$

Moreover, $l_{k}(\beta) \geq\left|a-a^{\prime}\right| / r$. Hence $2 l_{k}(\beta) \geq g(r)$ where

$$
g(r)=\frac{\left|a-a^{\prime}\right|}{r}+\log \frac{r}{1-t}+\log \frac{r}{1-t^{\prime}} .
$$


Since $\left|a-a^{\prime}\right| \geq 2$, we see by elementary calculus that $g$ is decreasing on $(0,1]$. Hence

$$
2 c l_{k}(\beta) \geq c g(1) \geq l(\alpha)+\log \frac{1}{1-t}+\log \frac{1}{1-t^{\prime}}=l_{k}\left(\gamma_{0}\right) .
$$

5.8. Lemma. There is a universal constant $c_{0}$ such that each pair $a, b \in G_{0}$ can be joined by an arc $\gamma$ that is $c_{0}$-quasiconvex both in the norm metric and in the $\mathrm{QH}$ metric of $G_{0}$.

Proof. If $\left|P_{1} a-P_{1} b\right| \geq 2$, the $\operatorname{arc} \gamma=\left[a, P_{1} a\right] \cup\left[P_{1} a, P_{1} b\right] \cup\left[P_{1} b, b\right]$ is a 2-neargeodesic of $G_{0}$ by 5.7. It is easy to verify that $\gamma$ is 2-quasiconvex in the norm metric.

Suppose that $\left|P_{1} a-P_{1} b\right|<2$, and set $z=\left(P_{1} a+P_{1} b\right) / 2$. Then $a$ and $b$ lie in the convex domain $D=B_{1}(z, 1) \times B_{2}$. Since $B(z, 1) \subset D \subset B(z, 2)$, the domain $D$ is 4-uniform by 2.19. Let $\gamma: a \curvearrowright b$ be a 2-neargeodesic of $D$. Applying the part $(1) \Rightarrow(2)$ of 2.42 with the substitution $G \mapsto D, D \mapsto G_{0}$ we see that $\gamma$ is a $c_{0^{-}}$ neargeodesic of $G_{0}$ with a universal $c_{0}$. Moreover by the Cigar theorem 2.29, each subarc of $\gamma$ is $c_{1}$-uniform in $D$ with a universal $c_{1}$, and hence $\gamma$ is $c_{1}$-quasiconvex in the norm metric.

5.9. Lemma. Let $F$ be a normed vector space with $\operatorname{dim} F \geq 2$, and let $B$ be a ball (open or closed) in $F$. Then each pair of points in $F \backslash B$ can be joined by a 5-quasiconvex arc in $F \backslash B$.

Proof. We may assume that $B$ is centered at the origin. Let $a, b \in F \backslash B$ with $|a|=r \leq R=|b|$. Setting $y=r b / R$ we have $|y|=r$. Since the sphere $S(r)$ is 2-quasiconvex by [Sc, $5 \mathrm{~F}]$, there is an $\operatorname{arc} \alpha: a \curvearrowright y$ in $S(r)$ with $l(\alpha) \leq 2|a-y|$. It suffices to show that $l(\gamma) \leq 5|a-b|$ for $\gamma=\alpha \cup[y, b]$. We have

$$
l(\gamma) \leq 2|a-y|+|y-b| \leq 2|a-b|+3|y-b| .
$$

Since $|y-b|=R-r \leq|a-b|$, we obtain $l(\gamma) \leq 5|a-b|$.

5.10. Proposition. For each $R>0$, the domain $G_{0}$ is $R$-boundedly $(R+2)$ uniform.

Proof. Let $a, b \in G_{0}$ with $|a-b| \leq R$. For $z=\left(P_{1} a+P_{1} b\right) / 2$, the domain $D=$ $G_{0} \cap B(z, 1+R / 2)$ is convex and contains $a$ and $b$. Moreover, $B(z, 1) \subset D \subset$ $B(z, 1+R / 2)$. Hence $D$ is $(R+2)$-uniform by 2.19 , and the proposition follows.

5.11. Proposition. The domain $G_{0}$ is locally 4-uniform and $(c, 0)$-LSC at each finite boundary point for all $c>1$. If $\operatorname{dim} E_{1} \geq 2$, then $G_{0}$ is $\left(c_{0}, 0\right)$-LSC at $\infty$ with a universal constant $c_{0}$.

Proof. Let $b=\left(b_{1}, b_{2}\right) \in \partial G_{0} \backslash\{\infty\}$, and set $D=B_{1}\left(b_{1}, 1\right) \times B_{2}=P_{1}^{-1} B_{1}\left(b_{1}, 1\right) \cap$ $G_{0}$. Then $B\left(b_{1}, 1\right) \subset D \subset B\left(b_{1}, 2\right)$, and hence $D$ is 4 -uniform by 2.19 . Consequently, $G_{0}$ is locally 4 -uniform at $b$. The LSC property follows from 4.3 and 5.10 .

Suppose that $\operatorname{dim} E_{2} \geq 2$. Let $R>0$ and let $x, y \in G_{0} \backslash\left(\bar{B}_{1}(R+2) \times B_{2}\right)$. It suffices to show that $x$ and $y$ can be joined by a $c_{0}$-neargeodesic of $G_{0}$ in $G_{0} \backslash$ $\left(\bar{B}_{1}(R) \times B_{2}\right)$. Writing $x=\left(x_{1}, x_{2}\right)$ and $y=\left(y_{1}, y_{2}\right)$ we consider two cases.

Case 1. $\left|x_{1}-y_{1}\right|<2$. The domain $D=B_{1}\left(x_{1}, 2\right) \times B_{2}$ is convex and 6-uniform by 2.19. Let $\gamma: x \curvearrowright y$ be a 2-neargeodesic of $D$. By 2.42, we again see that $\gamma$ is a $c_{0}$-neargeodesic of $G_{0}$ with a universal $c_{0}$. Moreover, $\gamma \subset G_{0} \backslash\left(\bar{B}_{1}(R) \times B_{2}\right)$. 
Case 2. $\left|x_{1}-y_{1}\right| \geq 2$. By 5.9 , we can join $x_{1}$ and $y_{1}$ by a 5 -quasiconvex arc $\alpha \subset E_{1} \backslash B_{1}(R)$. Then $\gamma=\left[x, x_{1}\right] \cup \alpha \cup\left[y_{1}, y\right]$ joins $x$ and $y$ in $G_{0} \backslash\left(B_{1}(R) \times B_{2}\right)$, and $\gamma$ is a 10 -neargeodesic by 5.7 .

5.12. Theorem. The domain $G_{0}$ is $\mathrm{CQH}$ equivalent to a uniform domain if and only if $\operatorname{dim} E_{1}=1$.

Proof. Suppose that $\operatorname{dim} E_{1}=1$, let $a \in E_{1}$ be a unit vector, and let $H$ be the half space $\left\{(t a, x): t>0, x \in E_{2}\right\}$ of $E=E_{1} \times E_{2}$. Then $H$ is a uniform domain, and the map $f: H \rightarrow G_{0}$, defined by

$$
f x=\frac{x}{|x|}+a \log \frac{1}{|x|},
$$

is 3-QH in the norm $|x|=\left|x_{1}\right|+\left|x_{2}\right|$ (see [Vä9, 8.14]).

Next assume that $\operatorname{dim} E_{2} \geq 2$ and that $f: G_{0} \rightarrow G$ is a CQH map onto a uniform domain $G$. Since $G_{0}$ and $G$ are LSC at all boundary points by 4.3 and 5.11 , and since $G_{0}$ is boundedly uniform by 5.10 , it follows from 4.8 that $f$ extends to a homeomorphism $\bar{f}: \bar{G}_{0} \rightarrow \bar{G}$. Choose a line $L \subset E_{1}$. Since $L$ is a geodesic of $G_{0}, f L$ is a solid open arc in $G$. Moreover, $f L \cup\{\bar{f}(\infty)\}$ is a Jordan curve. This is a contradiction by 4.15 .

5.13. Theorem. Suppose that $G_{0}=E_{1} \times B_{2}$ and $G_{0}^{\prime}=E_{1}^{\prime} \times B_{2}^{\prime}$ are domains as in 5.1 and that $f: G_{0} \rightarrow G_{0}^{\prime}$ is $\mathrm{CQH}$. Then $f$ has a limit at every finite boundary point of $G_{0}$, and $\operatorname{dim} E_{1}=\operatorname{dim} E_{1}^{\prime}, \operatorname{dim} E_{2}=\operatorname{dim} E_{2}^{\prime}$. If $\operatorname{dim} E_{1} \geq 2$, then $f$ extends to a homeomorphism $\bar{f}: \bar{G}_{0} \rightarrow \bar{G}_{0}^{\prime}$ with $\bar{f}(\infty)=\infty$.

Proof. Let $b \in \partial G_{0}, b \neq \infty$. Then $G$ is LSC at $b$ by 5.11 , and $G_{0}^{\prime}$ is boundedly uniform by 5.10 . Hence $f$ has a limit at $b$ by 4.8 .

If $\operatorname{dim} E_{1}=1$, then $\operatorname{dim} E_{1}^{\prime}=1$ by 5.12 , and hence $\operatorname{dim} E_{2}=\operatorname{dim} E_{2}^{\prime}$ (either both are infinite or both are finite and equal).

Suppose that $\operatorname{dim} E_{1} \geq 2$. Then $\operatorname{dim} E_{1}^{\prime} \geq 2$. Since $G_{0}$ and $G_{0}^{\prime}$ are now LSC also at $\infty$ by 5.11, $f$ extends to a homeomorphism $\bar{f}: \bar{G}_{0} \rightarrow \bar{G}_{0}^{\prime}$ by 4.8 . Let $L \subset E_{1}$ be a line. As in the proof of 5.12, $f L$ is an open solid arc in $G_{0}^{\prime}$, and $f L \cup\{\bar{f}(\infty)\}$ is a Jordan curve. From 4.15 and 5.11 it follows that $\bar{f}(\infty)=\infty$.

Since $\bar{f}$ defines a homeomorphism of $E_{1} \times \partial B_{2}$ onto $E_{1}^{\prime} \times \partial B_{2}^{\prime}$, the sets $\partial B_{2}$ and $\partial B_{2}^{\prime}$ are homotopy equivalent. Hence $\operatorname{dim} E_{2}=\operatorname{dim} E_{2}^{\prime}$, which implies that $\operatorname{dim} E_{1}=\operatorname{dim} E_{1}^{\prime}$.

5.14. Corollary. If $1 \leq p<q<n$, the domains $\mathrm{R}^{p} \times B^{n-p}$ and $\mathrm{R}^{q} \times B^{n-q}$ are not quasiconformally equivalent.

5.15. Remark. Consider the case $\operatorname{dim} E_{1}=1$ of 5.13. Replacing the one-point extension $\bar{G}_{0}=\left(E_{1} \times \bar{B}_{2}\right) \cup\{\infty\}$ by the two-point extension $G^{*}=\left(E_{1} \times \bar{B}_{2}\right) \cup$ $\{-\infty, \infty\}$ in the usual way, it is easy to see that $f$ extends to a homeomorphism $f^{*}: G^{*} \rightarrow G^{*}$. For example, one can make use of auxiliary QH maps of $G_{0}$ and $G_{0}^{\prime}$ onto half spaces; see the proof of 5.12 .

5.16. Conventions. In the rest of the paper we consider self maps of $G_{0}$. In all results henceforth we assume that:

(1) $G_{0}=E_{1} \times B_{2}$ as in 5.1.

(2) $f: G_{0} \rightarrow G_{0}$ is an $(M, C)$-CQH map.

(3) Either $\operatorname{dim} E_{1} \geq 2$ or $|f x| \rightarrow \infty$ as $|x| \rightarrow \infty$. 
From (3) it follows that $f$ extends to a homeomorphism $\bar{f}: \bar{G}_{0} \rightarrow \bar{G}_{0}$ with $\bar{f}(\infty)=$ $\infty$.

We estimate the vertical and horizontal distortions of $f$ in 5.17 and 5.18, and show that $f$ is coarsely bilipschitz in the norm metric in 5.19 . In 5.22 we prove a result called the Fundamental lemma, which is used in 5.24 to show that $\bar{f}$ is quasisymmetric rel $\partial G_{0}$. In 5.26 we prove that if $f$ is freely quasiconformal, then $f$ is quasisymmetric.

5.17. Theorem. If $a \in G_{0}$, then $\delta(f a) \geq \mu(\delta(a), M, C)>0$.

Proof. Set $r=\delta(a)$ and choose a line $L$ through $a$, parallel to $E_{1}$. Then $L$ is a $(1 / r)$-neargeodesic of $G_{0}$ by $5.4(5)$. Hence $f L$ is a $(c, h)$-solid arc with $(c, h)$ depending on $(r, M, C)$. By $5.10, G_{0}$ is 1-boundedly 3 -uniform and hence, by 2.27 , 1-boundedly $\mathrm{QH} \psi$-uniform with $\psi(t)=189 \log (1+t)$. Let $M_{1}=M_{1}(c, h, \psi)$ be the number given by $(2.11)$.

If $\delta(f a) \geq 1 / 4 M_{1}$, there is nothing to prove. Assume that $\delta(f a) \leq 1 / 4 M_{1}$. Since $\bar{f}(\infty)=\infty$, the arc $f L$ converges to $\infty$ at both ends. If $\delta(x)<1 / 4 M_{1}$ for all $x \in f L$, then $f L$ is bounded by the Escape lemma 2.13. Hence there is $x_{0} \in f L$ with $\delta\left(x_{0}\right) \geq 1 / 4 M_{1}$. Let $q=q(c, h, \psi)>0$ be the number given by the Diving lemma 2.15. If $\delta(x) \leq q \delta\left(x_{0}\right)$ for some $x \in f L$, then the $x$-component of $f L \backslash\left\{x_{0}\right\}$ is bounded by 2.15 . Hence $\delta(x)>q \delta\left(x_{0}\right)$ for all $x \in f L$, and the theorem holds with $\mu=q / 4 M_{1}$.

5.18. Theorem. If $a, b \in G_{0}$ with $P_{1} a=P_{1} b$, then $|f a-f b| \leq c_{1}(M, C)$.

Proof. By the triangle inequality we may assume that $P_{2} a=0$. Set $x=f^{-1} P_{1} f b$, $\gamma^{\prime}=[f x, f b], \gamma=f^{-1} \gamma^{\prime}$. Then $\gamma^{\prime}$ is a QH geodesic, and hence $\gamma$ is $(c, h)$-solid with $(c, h)$ depending on $(M, C)$. Let $M_{1}=M_{1}(c, h, \psi)$ be as in the preceding proof, and set $r=1 / 5 M_{1}$. Since $\delta(f x)=1,5.17$ gives $\delta(x) \geq \mu=\mu(1, M, C)>0$. We consider three cases.

Case 1. $|b-x| \leq 1$. Now $|a-x| \leq|a-b|+|b-x| \leq 2$, and hence

$$
\begin{aligned}
|f a-f b| & \leq|f a-f x|+1 \leq k(f a, f x)+1 \leq M k(a, x)+C+1 \\
& \leq M|a-x| / \mu+C+1 \leq 2 M / \mu+C+1 .
\end{aligned}
$$

Case 2. $\delta(b) \geq r$. Now $k(a, b) \leq|a-b| / r \leq 1 / r=5 M_{1}$, and hence

$$
|f a-f b| \leq k(f a, f b) \leq M k(a, b)+C \leq 5 M M_{1}+C .
$$

Case 3. $|b-x| \geq 1$ and $\delta(b) \leq r$. If $z \in \gamma$ and if $\gamma[b, z] \subset \partial G_{0}+\bar{B}(r)$, then the Escape lemma 2.13 gives

$$
|b-z| \leq 4 M_{1} r=4 / 5 \leq|b-x| .
$$

Hence there is the first point $z$ of $\gamma$ such that $\delta(z)=r$. Since $|a-z| \leq|a-b|+|b-z|<$ 2 , we get

$$
k(a, z) \leq|a-z| / r<2 / r=10 M_{1},
$$

which yields

$$
\begin{aligned}
|f a-f b| & \leq|f a-f z|+1 \leq k(f a, f z)+1 \\
& \leq M k(a, z)+C+1 \leq 10 M M_{1}+C+1 .
\end{aligned}
$$


5.19. Theorem. The map $f: G_{0} \rightarrow G_{0}$ is $C_{1}$-coarsely $M$-bilipschitz in the norm metric with $C_{1}=C_{1}(M, C)$. That is,

$$
\left(|x-y|-C_{1}\right) / M \leq|f x-f y| \leq M|x-y|+C_{1}
$$

for all $x, y \in G_{0}$.

Proof. Let $x=\left(x_{1}, x_{2}\right), y=\left(y_{1}, y_{2}\right) \in G_{0}$. By 5.18 we obtain

$$
\begin{aligned}
|f x-f y| & \leq\left|f x-f x_{1}\right|+\left|f x_{1}-f y_{1}\right|+\left|f y_{1}-f y\right| \leq k\left(f x_{1}, f y_{1}\right)+2 c_{1} \\
& \leq M k\left(x_{1}, y_{1}\right)+C+2 c_{1} \leq M|x-y|+C+2 c_{1} .
\end{aligned}
$$

Since $f^{-1}$ is $(M, C)$-CQH, the theorem follows.

5.20. Quasisymmetry. We recall the basic definitions of quasisymmetry. Let $\eta:[0, \infty) \rightarrow[0, \infty)$ be a homeomorphism. By a triplet in a metric space $(X, \varrho)$ we mean a triple $T=(x, y, z)$ of distinct points in $X$, and we write

$$
|T|=\varrho(x, y) / \varrho(x, z) .
$$

An embedding $f: X \rightarrow Y$ into a metric space $Y$ is $\eta$-quasisymmetric if $|f T| \leq \eta(|T|)$ for each triplet $T$ in $X$. If $\dot{X}=X \cup\{\infty\}$ is the one-point extension of $X$ and if $f: \dot{X} \rightarrow \dot{Y}$ is an embedding such that $f(\infty)=\infty$ and $f$ defines an $\eta$-quasisymmetric map $f: X \rightarrow Y$, we also say that $f$ is $\eta$-quasisymmetric.

If $A \subset X$, a triplet $(x, y, z)$ in $X$ is said to be a triplet in $(X, A)$ if either $x \in A$ or $\{y, z\} \subset A$. An embedding $f: X \rightarrow Y$ is $\eta$-quasisymmetric rel $A$ if $|f T| \leq \eta(|T|)$ for each triplet $T$ in $(X, A)$.

We want to show that the map $\bar{f}: \bar{G}_{0} \rightarrow \bar{G}_{0}$ is quasisymmetric rel $\partial G_{0}$. The corresponding result for uniform domains was given in [Vä6, 7.9]. The proof was based on [Vä6, Fundamental lemma 7.3]. We give a variation of the latter result in 5.22. First we prove a simple consequence of 5.19 .

5.21. Lemma. Let $T=(x, y, z)$ be a triplet in $G_{0}$ with $|x-z| \geq 2 C_{1}$, where $C_{1}$ is given by (5.19). Then $|f T| \leq 2 M^{2}|T|+M$.

Proof. By 5.19 we get

$$
\begin{aligned}
& |f x-f y| \leq M|x-y|+C_{1} \leq M|T||x-z|+|x-z| / 2, \\
& |f x-f z| \geq\left(|x-z|-C_{1}\right) / M \geq|x-z| / 2 M
\end{aligned}
$$

and the lemma follows.

5.22. Fundamental lemma. Suppose that $T=(x, y, z)$ is a triplet in $G_{0}$ such that $|T| \leq 1$ and $k(x, z) \geq 2 C \vee \frac{1}{2}$. Then $|f T| \leq H(M, C)$.

Proof. Let $C_{1}=C_{1}(M, C)$ be the number given by 5.19. If $|x-z| \geq 2 C_{1}$, the result follows from 5.21. Hence we may assume that $|x-z| \leq 2 C_{1}$.

Write $|x-z|=t,|f x-f z|=t^{\prime}$. We want to find an estimate $|f x-f y| \leq H t^{\prime}$. Let $L_{0} \subset E_{1}$ be a line containing $P_{1} f x$ and $P_{1} f y$, and let $L$ be a component of $L_{0} \backslash\left\{P_{1} f y\right\}$, not containing $P_{1} f x$. The $\operatorname{arc} \alpha^{\prime}=\left[f y, P_{1} f y\right] \cup L$ is a 2-neargeodesic of $G_{0}$ by 5.5 .

By 5.8 there is an arc $\beta^{\prime}: f x \curvearrowright f z$, which is $c_{0}$-quasiconvex both in the norm metric and in the QH metric of $G_{0}$ with a universal constant $c_{0}$. The $\operatorname{arcs} \alpha=f^{-1} \alpha^{\prime}$ and $\beta=f^{-1} \beta^{\prime}$ are $(c, h)$-solid with $(c, h)$ depending on $(M, C)$.

By $5.10, G_{0}$ is $2 C_{1}$-boundedly $\left(2 C_{1}+2\right)$-uniform, and hence, by $2.27,2 C_{1}$ boundedly $\mathrm{QH} \psi$-uniform with a slow function $\psi$ depending only on $(M, C)$. Let 
$M_{1}=M_{1}(c, h, \psi)$ be the number given by $(2.11)$, and set $r=t / 5 M_{1}$. Let $z_{1}$ be the first point of $\beta$ with $\left|x-z_{1}\right|=t$, and set $\beta_{0}=\beta\left[x, z_{1}\right]$. Let $y_{1}$ be the first point of $\alpha$ with $\left|y-y_{1}\right|=t$, and set $\alpha_{0}=\alpha\left[y, y_{1}\right]$.

Fact 1. $\alpha_{0} \not \subset \partial G_{0}+\bar{B}(r)$ and $\beta_{0} \not \subset \partial G_{0}+\bar{B}(r)$.

Assume that $\alpha_{0} \subset \partial G_{0}+\bar{B}(r)$. Since $r \leq 2 C_{1} / 5 M_{1}$, the Escape lemma 2.13 implies that $d\left(\alpha_{0}\right) \leq 4 M_{1} r=4 t / 5<t$. Since $d\left(\alpha_{0}\right) \geq\left|y-y_{1}\right|=t$, this is a contradiction. The second part is proved similarly. Then

By Fact 1 we can choose points $y_{0} \in \alpha_{0}$ and $z_{0} \in \beta_{0}$ such that $\delta\left(y_{0}\right) \wedge \delta\left(z_{0}\right) \geq r$.

$$
\left|y_{0}-z_{0}\right| \leq\left|y_{0}-y\right|+|y-x|+\left|x-z_{0}\right| \leq 3 t .
$$

Hence $k\left(y_{0}, z_{0}\right) \leq\left|y_{0}-z_{0}\right| / r \leq 3 t / r=15 M_{1}$, which yields

$$
k\left(f y_{0}, f z_{0}\right) \leq 15 M M_{1}+C=c_{1}
$$

with $c_{1}=c_{1}(M, C)$.

Fact 2. $\delta(f x) \leq 8 M t^{\prime}$.

Assume that $\delta(f x)>8 M t^{\prime}$. Since $\delta(f x)>2 t^{\prime}=2|f x-f z|$, we have $k(f x, f z) \leq$ $2 t^{\prime} / \delta(f x)<1 / 4 M$, and we get the contradiction

$$
1 / 4+C \leq(2 C) \vee(1 / 2) \leq k(x, z) \leq M k(f x, f z)+C<1 / 4+C .
$$

Fact 3. $|f x-f y| \leq\left(32 M t^{\prime}\right) \vee 6\left|f x-f y_{0}\right|$.

We assume that $|f x-f y|>32 M t^{\prime}$ and show that $|f x-f y| \leq 6\left|f x-f y_{0}\right|$. We consider two cases.

Case 1. $\left|f y_{0}-P_{1} f y\right| \leq 1$. If $f y_{0} \in\left[f y, P_{1} f y\right]$, then $\left|f y_{0}-f y\right|<\delta\left(f y_{0}\right)$. If $f y_{0} \in L$, then $\left|f y_{0}-f y\right| \leq\left|f y_{0}-P_{1} f y\right|+\left|P_{1} f y-f y\right| \leq 2=2 \delta\left(f y_{0}\right)$, and thus we always have $\left|f y_{0}-f y\right| \leq 2 \delta\left(f y_{0}\right)$. Since Fact 2 gives

$$
\delta\left(f y_{0}\right) \leq \delta(f x)+\left|f x-f y_{0}\right| \leq 8 M t^{\prime}+\left|f x-f y_{0}\right| \leq|f x-f y| / 4+\left|f x-f y_{0}\right|,
$$

we obtain

$$
|f x-f y| \leq\left|f x-f y_{0}\right|+\left|f y_{0}-f y\right| \leq|f x-f y| / 2+3\left|f x-f y_{0}\right|,
$$

and hence $|f x-f y| \leq 6\left|f x-f y_{0}\right|$.

Case 2. $\left|f y_{0}-P_{1} f y\right| \geq 1$. Now $P_{1} f y \in\left[P_{1} f x, f y_{0}\right] \subset L_{0}$, and hence

$$
\begin{aligned}
|f x-f y| & \leq\left|P_{1} f x-P_{1} f y\right|+2 \leq 2\left|P_{1} f x-P_{1} f y\right|+2\left|P_{1} f y-f y_{0}\right| \\
& =2\left|P_{1} f x-f y_{0}\right| \leq 2\left|f x-f y_{0}\right|,
\end{aligned}
$$

and Fact 3 is proved.

We turn to the proof for the estimate $|f x-f y| \leq H t^{\prime}$. If $|f x-f y| \leq 32 M t^{\prime}$, it holds with $H=32 M$. By Fact 3 we may thus assume that $|f x-f y| \leq 6\left|f x-f y_{0}\right|$. Using [Vä5, 2.2(1)], (5.23), Fact 2, and the $c_{0}$-quasiconvexity of $\beta^{\prime}$ we obtain

$$
\begin{aligned}
\left|f x-f y_{0}\right| & \leq\left|f y_{0}-f z_{0}\right|+\left|f x-f z_{0}\right| \leq \delta\left(f z_{0}\right) e^{k\left(f y_{0}, f z_{0}\right)}+\left|f x-f z_{0}\right| \\
& \leq\left(\delta(f x)+\left|f x-f z_{0}\right|\right) e^{c_{1}}+\left|f x-f z_{0}\right| \\
& \leq 8 M e^{c_{1}} t^{\prime}+\left(e^{c_{1}}+1\right) c_{0} t^{\prime}=c_{2} t^{\prime}
\end{aligned}
$$

with $c_{2}=c_{2}(M, C)$. Hence $|f x-f y| \leq 6 c_{2} t^{\prime}$.

Recall that in the following results, we assume that $f$ and $G_{0}$ satisfy the conditions of 5.16 . 
5.24. Theorem. The map $\bar{f}: \bar{G}_{0} \rightarrow \bar{G}_{0}$ is $\eta$-quasisymmetric rel $\partial G_{0}$ with $\eta$ depending only on $(M, C)$.

Proof. Let $T=(x, y, z)$ be a triplet in $\left(\bar{G}_{0} \backslash\{\infty\}, \partial G_{0} \backslash\{\infty\}\right)$. By symmetry and by $[\mathrm{Vä6}, 5.8]$, it suffices to find an estimate $|f T| \leq \eta(|T|)$, where $\eta:(0, \infty) \rightarrow(0, \infty)$ is increasing, but $\eta(t)$ need not converge to 0 as $t \rightarrow 0$.

Choose a sequence of triplets $T_{n}=\left(x_{n}, y_{n}, z_{n}\right)$ in $G_{0}$ such that $x_{n} \rightarrow x, y_{n} \rightarrow$ $y, z_{n} \rightarrow z$. Write $R=2 C \vee \frac{1}{2}$. Since $\delta\left(x_{n}\right) \rightarrow 0$ or $\delta\left(z_{n}\right) \rightarrow 0$, we may assume that $\left|x_{n}-z_{n}\right| \geq e^{R}\left(\delta\left(x_{n}\right) \wedge \delta\left(z_{n}\right)\right)$ for all $n$. This implies that $k\left(x_{n}, z_{n}\right) \geq R$. Fix $n$ and choose successive points $x_{n}=a_{0}, \ldots, a_{N}=y_{n}$ of the line segment $\left[x_{n}, y_{n}\right] \subset G_{0}$ such that $a_{1}$ is the last point with $\left|a_{1}-x_{n}\right| \leq\left|x_{n}-z_{n}\right|$, and for $j \geq 2, a_{j}$ is the last point with $\left|a_{j}-a_{j-1}\right| \leq\left|a_{j-1}-x_{n}\right|$. By the Fundamental lemma 5.22 we have $\left|f a_{1}-f x_{n}\right| \leq H\left|f x_{n}-f z_{n}\right|$ with a constant $H=H(M, C)$. If $N=1$, this gives $\left|f T_{n}\right| \leq H$. If $N \geq 2$, the Fundamental lemma gives by induction

$$
\begin{aligned}
\left|f a_{j+1}-f x_{n}\right| & \leq\left|f a_{j+1}-f a_{j}\right|+\left|f a_{j}-f x_{n}\right| \leq(H+1)^{j}\left|f a_{1}-f x_{n}\right| \\
& \leq H(H+1)^{j}\left|f x_{n}-f z_{n}\right|
\end{aligned}
$$

for $1 \leq j \leq N-1$, and hence $\left|f T_{n}\right| \leq H(H+1)^{N-1}$. Since

$$
\left|x_{n}-y_{n}\right|=\sum_{j=1}^{N}\left|a_{j-1}-a_{j}\right| \geq(N-1)\left|a_{1}-x_{n}\right|=(N-1)\left|x_{n}-z_{n}\right|,
$$

this implies that $\left|f T_{n}\right| \leq H(H+1)^{\left|T_{n}\right|}$. As $n \rightarrow \infty$, this gives the desired estimate $|f T| \leq \eta(|T|)$ with $\eta(t)=H(H+1)^{t}$.

5.25. Corollary. The induced map $\partial G_{0} \rightarrow \partial G_{0}$ is $\eta$-quasisymmetric with $\eta$ depending only on $(M, C)$.

5.26. Theorem. If $f$ is freely quasiconformal, then $f$ is $\eta$-quasisymmetric with $\eta=\eta_{\varphi}$

Proof. Since $G_{0}$ is convex, it suffices to show that $f$ is weakly $H$-quasisymmetric with $H=H(\varphi)$ (see [Vä6, 5.5]). Let $T=(x, y, z)$ be a triplet in $G_{0}$ with $|T| \leq 1$. We must find an estimate $|f T| \leq H(\varphi)$.

By [Vä6, 4.14], $f$ is $(M, 1 / 4)$-CQH with some $M=M(\varphi)$. If $k(x, z) \geq 1 / 2$, the desired estimate follows from the Fundamental lemma 5.22. If $k(x, z) \leq 1 / 2$, then

$$
|x-y| / \delta(x) \leq e^{k(x, y)}-1 \leq \sqrt{e}-1=q<1 .
$$

Since $f$ is $q$-locally $\eta_{\varphi}$-quasisymmetric by $[\mathrm{V} a ̈ 5,5.10]$, we have $|f T| \leq \eta(1)$.

5.27. Corollary. If $f: \mathrm{R}^{p} \times B^{q} \rightarrow \mathrm{R}^{p} \times B^{q}$ is $K$-quasiconformal and if $p \geq 2$, then $f$ is $\eta$-quasisymmetric with $\eta=\eta_{K, p, q}$.

\section{REFERENCES}

[Al] P. Alestalo, Quasisymmetry in product spaces and uniform domains, Licentiate's thesis, University of Helsinki, 1991 (Finnish).

[BV1] Z. Balogh and A. Volberg, Geometric localization, uniformly John property and separated semihyperbolic dynamics, Ark. Mat. 34, 1996, 21-49. MR 97i:30033

[BV2] _ Boundary Harnack principle for separated semihyperbolic repellers, harmonic measure applications, Rev. Mat. Iberoamericana 12, 1996, 299-336. MR 97m:31001

[BHK] M. Bonk, J. Heinonen and P. Koskela, Uniformizing Gromov hyperbolic spaces (in preparation). 
[FHM] J.L. Fernández, J. Heinonen and O. Martio, Quasilines and conformal mappings, J. Analyse Math. 52, 1989, 117-132. MR 90a:30017

[GO] F.W. Gehring and B.G. Osgood, Uniform domains and the quasihyperbolic metric, J. Analyse Math. 36, 1979, 50-74. MR 81k:30023

[GP] F.W. Gehring and B.P. Palka, Quasiconformally homogeneous domains, J. Analyse Math. 30, 1976, 172-199. MR 55:10676

[Jo1] P.W. Jones, Extension theorems for BMO, Indiana Univ. Math. J. 29, 1980, 41-66. MR 81b: 42047

[Jo2] _ Quasiconformal mappings and extendability of functions in Sobolev spaces, Acta Math. 147, 1981, 71-88. MR 83i:30014

[Ma] O. Martio, Definitions for uniform domains, Ann. Acad. Sci. Fenn. Math. 5, 1980, 197-205. MR 82c: 30028

[MaS] O. Martio and J. Sarvas, Injectivity theorems in plane and space, Ann. Acad. Sci. Fenn. Math. 4, 1979, 383-401. MR 81i:30039

[MiS] V. D. Milman and G. Schechtman, Asymptotic theory of finite-dimensional normed spaces, Lecture Notes in Mathematics 1200, Springer-Verlag, 1986. MR 87m:46038

[Sc] J.J. Schäffer, Geometry of spheres in normed spaces, Marcel Dekker, 1976. MR 57:7120

[Th] W. P. Thurston, The geometry and topology of three-manifolds, Mimeographed notes, Princeton University, 1980.

[Vä1] J. Väisälä, Lectures on $n$-dimensional quasiconformal mappings, Lecture Notes in Mathematics 229, Springer-Verlag, 1971. MR 56:12260

[Vä2] — Quasimöbius maps, J. Analyse Math. 44, 1984/85, 218-234. MR 87f:30059

[Vä3] Uniform domains, Tôhoku Math. J. 40, 1988, 101-118. MR 89d:30027

[Vä4] Q Quasiconformal maps of cylindrical domains, Acta Math. 162, 1989, 201-225. MR 90f:30034

[Vä5] , Free quasiconformality in Banach spaces. I, Ann. Acad. Sci. Fenn. Math. 15, 1990, 355-379. MR 92d:30012

[Vä6] - Free quasiconformality in Banach spaces. II, Ann. Acad. Sci. Fenn. Math. 16, 1991, 255-310. MR 94c:30028

[Vä7] , Free quasiconformality in Banach spaces. III, Ann. Acad. Sci. Fenn. Math. 17, 1992, 393-408. MR 94c:30029

[Vä8] $\quad$ Free quasiconformality in Banach spaces IV, Analysis and Topology, ed. by C. Andreian Cazacu et al., World Scientific (to appear).

[Vä9] _ The free quasiworld, Proceedings of the fifth Finnish-Polish-Ukranian summer school in complex analysis in Lublin 1996 (to appear).

Matematilkan laitos, Helsingin yliopisto, PL 4, Yliopistonkatu 5, 00014 Helsinki, FinLAND

E-mail address: jvaisala@cc.helsinki.fi 